FERMILAB-TM-2527-DI

November 2011

\title{
Accelerator/Experiment Operations - FY 2011
}

\author{
P. Adamson, G. Bernardi, M. Casarsa, R. Coleman, D. Denisov, R. Dixon, G. Ginther, \\ S. Gruenendahl, S. Hahn, D. Harris, S. Henderson, W. Kissel,W. M. Lee, K. McFarland, \\ A. Mitra, C. Moore, B. Pahlka, R. Plunkett, E. Ramberg, P. Schlabach,
}

A. K. Soha, R. Van de Water

Edited by J. A. Appel and H. Ramamoorthi

This Technical Memorandum (TM) summarizes the Fermilab accelerator and accelerator experiment operations for FY 2011. It is one of a series of annual publications intended to gather information in one place. In this case, the information concerns the FY 2011 Run II at the Tevatron Collider, the MINOS and MINERvA experiments using the Main Injector Neutrino Beam (NuMI), the MiniBooNE experiment running in the Booster Neutrino Beam (BNB), and the Meson Test Beam (MTest) activities in the $120 \mathrm{GeV}$ external Switchyard beam (SY120).

Each section was prepared by the relevant authors, and was somewhat edited for inclusion in this summary.

Accelerator Operations (R. Dixon, S. Henderson)

\section{Tevatron Collider}

The Tevatron performed well as FY 2011 began. Monthly integrated luminosity accumulations were averaging near $250 \mathrm{pb}^{-1}$ per month. There were a number of problems in the Pelletron including high voltage regulation and collector problems that persisted into the winter months. Nevertheless, the milestone of delivery of $10 \mathrm{fb}^{-1}$ of integrated luminosity was achieved for both the CDF and DZero experiments in the middle of December, 2010.

On December 25 a vacuum problem developed after a quench at B1, and the subsequent repair took approximately two weeks, and was completed over the holiday period. Another major interruption to Tevatron operations occurred in March. A lightning strike resulted in a failed magnet and spool piece.

Record peak and integrated luminosities were achieved in the spring. Tevatron parameters had been optimized to achieve these goals. Optimization of the initial luminosities for data taking resulted in slightly lower peak for the remainder of the year. Late in the summer there was an off-site power glitch and more voltage regulation problems in the Pelletron, resulting in significant downtime for the Collider program. Nevertheless, the Tevatron continued to run at near record levels until its shutdown on September 30. The integrated luminosity for the year was a record $2567 \mathrm{pb}^{-1}$. At the end of the running period, a week was dedicated to taking low energy data in CDF and DZero. This was done to map the collider results onto other world data. 
At 2:36 p.m. on Friday, September 30, 2011, the Tevatron was formally turned off for the final time. During the last four-week reporting period, the Tevatron ran well. During the last full 168 hour period from Friday, September 23 at 07:00 to Friday, September 30 at 07:00 the Tevatron delivered an integrated $75.447 \mathrm{pb}^{-1}$, for a fast sprint to the end of 28 years of Tevatron operations.

During the final summer of the Tevatron program, a series of studies was undertaken to document the machine, and to provide useful information to the LHC and other accelerator efforts in the world. Among the studies completed were a set of crystal-collimator studies and a series of hollow-beam studies using the Tevatron Electron Lens. Both of these studies have implications for the LHC. The studies required dedicated study periods that were interspersed with Collider running.

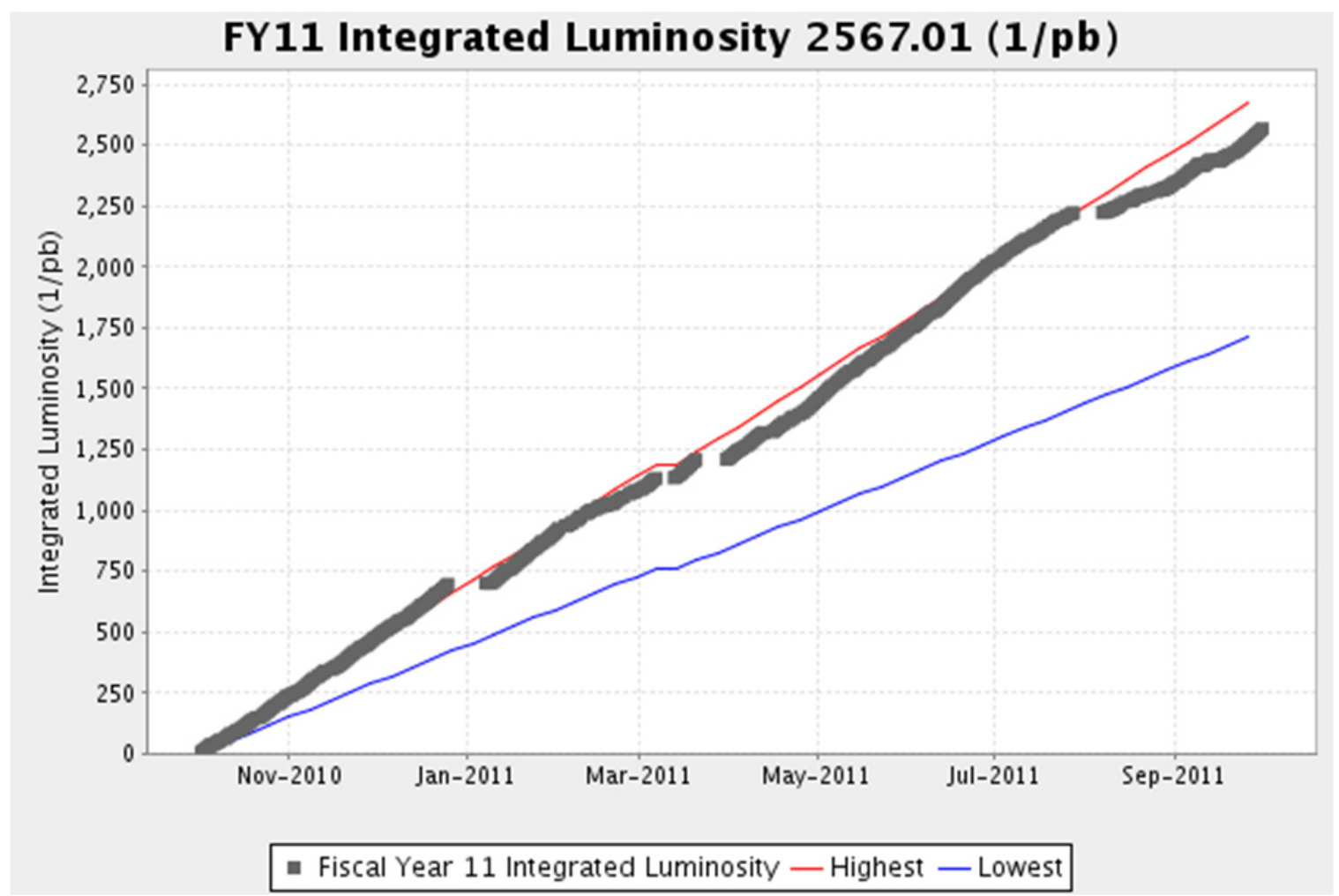

Figure 1. FY 2011 integrated luminosity, planned and delivered (average of CDF and DZero).

Final Status Relative to the FY 2011 Tevatron Plan

Table I shows the planned and actual performance of the Tevatron for the year (defined as from the first Monday of the fiscal year through the last day of Tevatron operations on September 30, 2011. 
Table I. Tevatron Collider planned and actual performance in the Fiscal Year 2011. Note that the Base and Design Profiles given here are the final ones, and are increased goals developed after the start of the fiscal year.

\begin{tabular}{|l|l|l|l|}
\hline & Base Profile & Design Profile & Actual $^{*}$ \\
\hline Median Init. Luminosity $\left(\mathrm{cm}^{-2} \mathrm{~s}^{-1}\right)$ & $2.1 \times 10^{32}$ & $3.5 \times 10^{32}$ & $3.40 \times 10^{32} *$ \\
\hline Protons/bunch & $265 \times 10^{9}$ & $295 \times 10^{9}$ & $270 \times 10^{9} *$ \\
\hline Antiprotons/bunch & $47 \times 10^{9}$ & $104 \times 10^{9}$ & $80.4 \times 10^{9 *}$ \\
\hline Accumulator-Recycler transfer eff. & $91 \%$ & $96.5 \%$ & $95 \%$ \\
\hline Typical Peak Stacking rate (mA/hour) & 26 & 31 & 25 \\
\hline FY11 integrated luminosity to date $\left(\mathrm{pb}^{-1}\right)$ & 1734 & 2708 & 2546 \\
\hline FY11 integrated store hours to date & 5100 & 6120 & 5424 \\
\hline FY11 scheduled uptime to date (hours) & & & 8177 \\
\hline $\begin{array}{l}\text { FY11 unscheduled downtime to date } \\
\text { (hours) }\end{array}$ & & $\begin{array}{l}1592 \\
(19.5 \%)\end{array}$ \\
\hline
\end{tabular}

* "Base" and "Design" labels correspond to "end-of-year" goals extended, where appropriate, to the standard reporting periods (Mondays through Sundays). "Actual" values in the first three rows correspond to median values over the entire fiscal year. The goals for Base/Design integrated luminosity per week were $32.2 / 52.1 \mathrm{pb}^{-1}$, and store hours of $100 / 120$ hours/week, for fully scheduled weeks.

\section{FY 2011 Neutrino Operations}

Early in 2011 the low-energy and high-energy neutrino beams were running steadily. Nevertheless, a helium leak into the NuMI target water system was detected, portending problems that would soon materialize with the failure of the target in February. Target replacement overlapped with down time at the Soudan Mine, where there was a fire in the access shaft leading to the CDMS and MINOS experiments. The NuMI replacement target was a new target that had been modified to strengthen the water circuit inside the target canister in an attempt to have a more robust target. NuMI operations resumed in early April. It took only 3 days for a new leak to develop. In order to reduce the rate at which the leak was growing, the beam intensity on target was reduced from over $3 \times 10^{16}$ to $1.8 \times 10^{16}$ protons on target per pulse. In spite of the reduced intensity the target failed in the middle of May. NT-01, which had run for more than a year previously and was replaced due to a failed motion drive on the carriage, was used to replace the failed target. Meanwhile careful analysis of the target issues was underway, and several design changes were implemented for the new spare targets. Beam resumed in June, but water began leaking into the target canister later in the month. In early July target scans revealed that there was water in the target canister. Since the spare tartest were not yet ready for installation, NT-02, the most durable target in previous running, was put back in. It had run for nearly 3 years without failing, and was finally replaced due to the degradation of the target material. Operations resumed in early August. In the middle of September the NuMI beam was shutdown to replace the old NT-02 target with the modified new spare target, NT-07. It continues to perform to the present time. 
It is important to note that during February, a new Main Injector intensity record of $46.9 \times 10^{12}$ protons per pulse ( $401 \mathrm{KW}$ average beam power) was achieved. These records were obtained with eleven-batch slip-stacking. The achievement was the culmination of work including rf upgrades, beam collimators, and the installation and commissioning of the gap clearing kickers. This achievement portends well for NOvA and LBNE running.

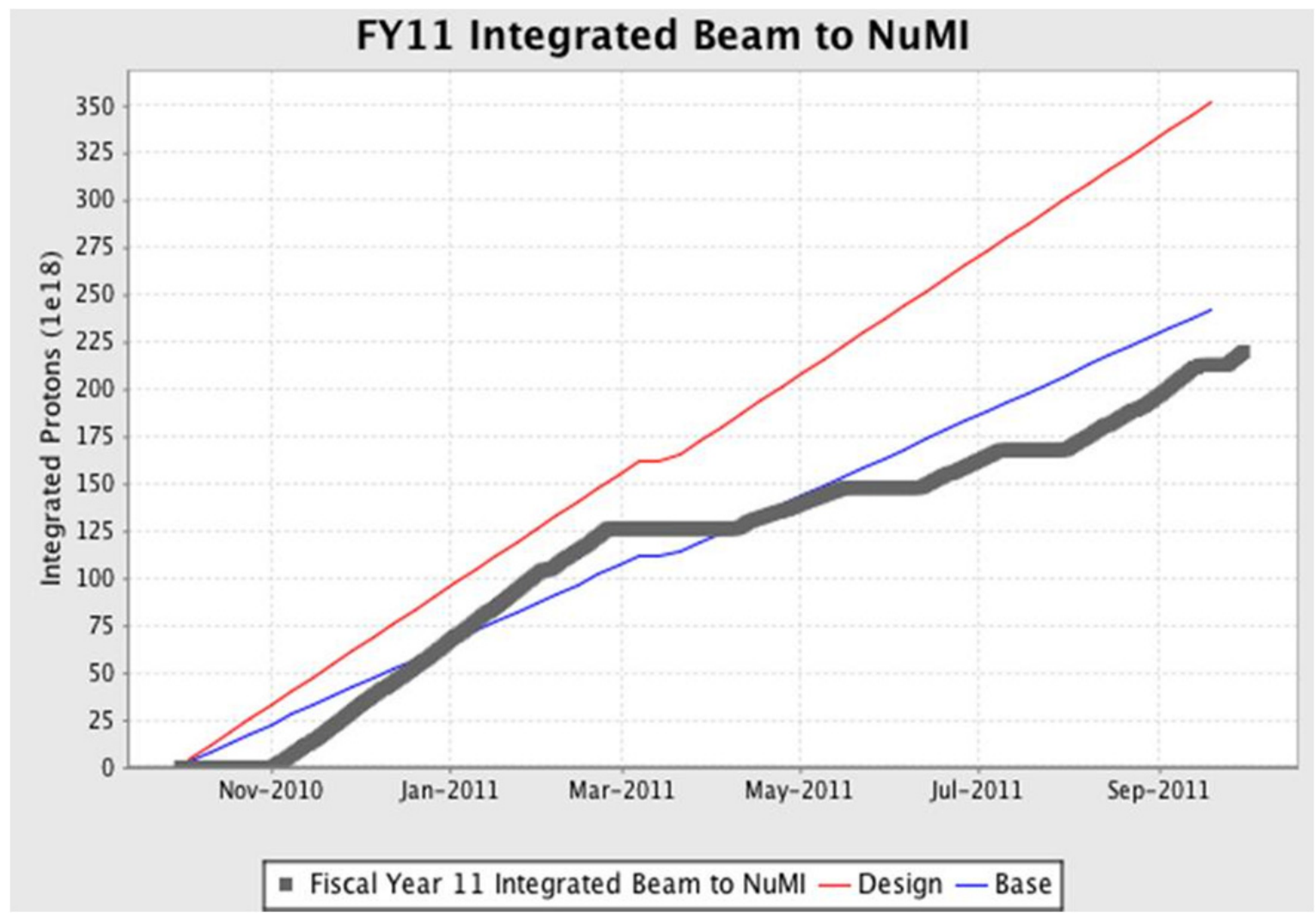

Figure 2. Fiscal Year 2011 integrated proton beam for NuMI.

MiniBooNE took advantage of the NuMI target incidents and improved Booster performance to accumulate $3.25 \times 10^{20}$ Protons, which was well above the expectation for the year, and was a record. This is a $91 \%$ increase over the previous year. They ran in the antineutrino mode for the entire year. 


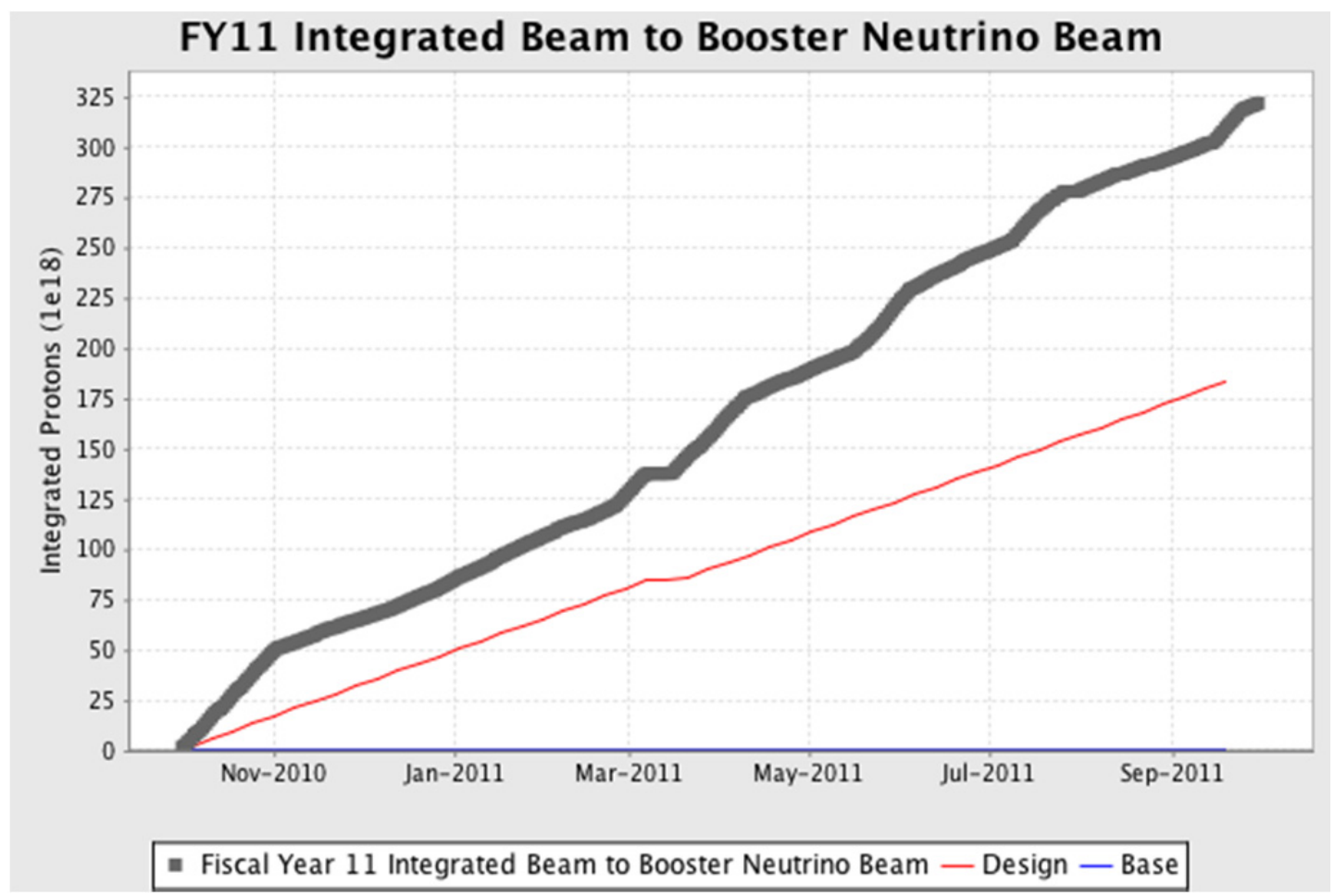

Figure 3. Fiscal year 2011 integrated proton beam for the Booster Neutrino Beam.

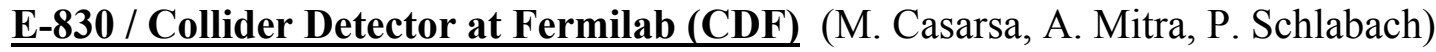

During FY 2011 a total integrated luminosity of $2.6 \mathrm{fb}^{-1}$ was delivered to the CDF detector, of which $2.2 \mathrm{fb}^{-1}$ was recorded to tape. At the end of FY 2011, a total of $12.0 \mathrm{fb}^{-1}$ had been delivered to the experiment in Run II (Fig. 4). Overall, the CDF detector operated with good live-time and no significant issues, even at the highest instantaneous luminosities. The total data-collection efficiency was $83 \%$, including dead time associated with trigger acceptance, operational inefficiencies (e.g. starting and stopping runs), and downtime from detector problems. 


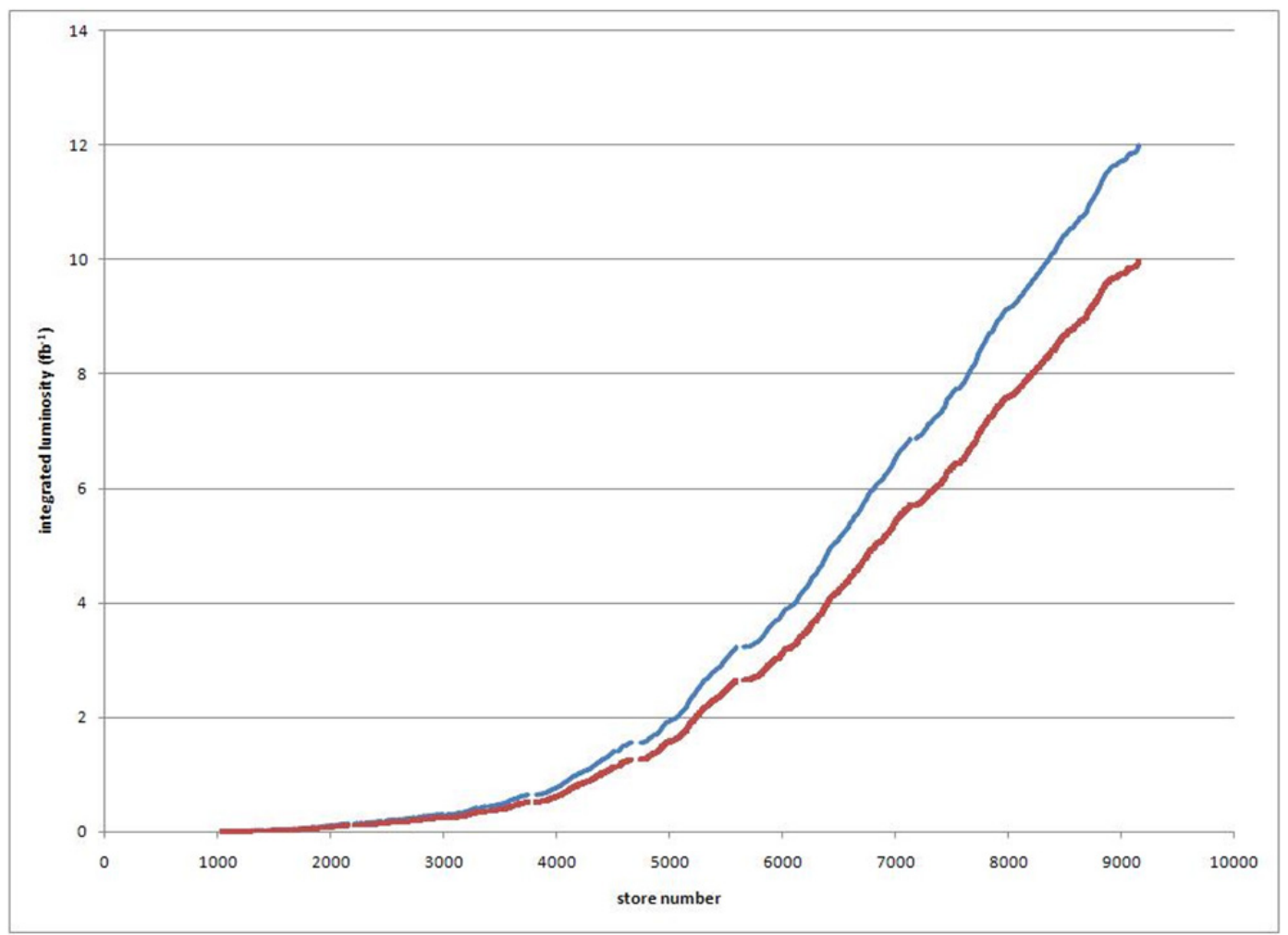

Figure 4. Delivered and recorded luminosity at CDF in Run II.

The Tevatron initial instantaneous luminosities were steadily around $3 \times 10^{32} \mathrm{~cm}^{-2} \mathrm{sec}^{-1}$. The median, average, and maximum were $3.2,2.9$, and $4.3 \times 10^{32} \mathrm{~cm}^{-2} \mathrm{sec}^{-1}$, respectively. Operating at the highest luminosity required several changes. As usual the CDF trigger was adjusted to cope with the higher instantaneous luminosities. CDF also changed to a "hit counting" algorithm for calculating the instantaneous luminosity for luminosities above $4 \times 10^{32}$ $\mathrm{cm}^{-2} \mathrm{sec}^{-1}$. No additional changes to detector operation were required. In particular, the high voltage systems for the central tracker worked fine at the highest luminosities. The silicon detector also continued to age as the total delivered luminosity increased. To compensate CDF changed detector parameters over time to maintain high efficiency and low noise-hit levels.

As can be seen in Fig. 5, there was only a brief shutdown during this fiscal year. During the one week shutdown CDF opened the detector bore for maintenance of the central tracking chamber high voltage. CDF also replaced the large purge fan for the Collision Hall HVAC. CDF returned quickly to efficient running after the shutdown. There were several other unplanned interruptions to data taking of about a week. In November, a wire in superlayer 5 of the COT broke and shorted out a quadrant of layers 5-8. This required a 2 day access to open the end plug, break the gas seal on the face of the detector, fish the wire out, and re-establish the seal. Including the time to inert the chamber with nitrogen, and then to refill with argon-ethane, CDF took no useful data for 5 days. Note that this was the third such incident, the first since 2002. The other downtimes were not specific to CDF: power outages, magnet replacements, etc. 


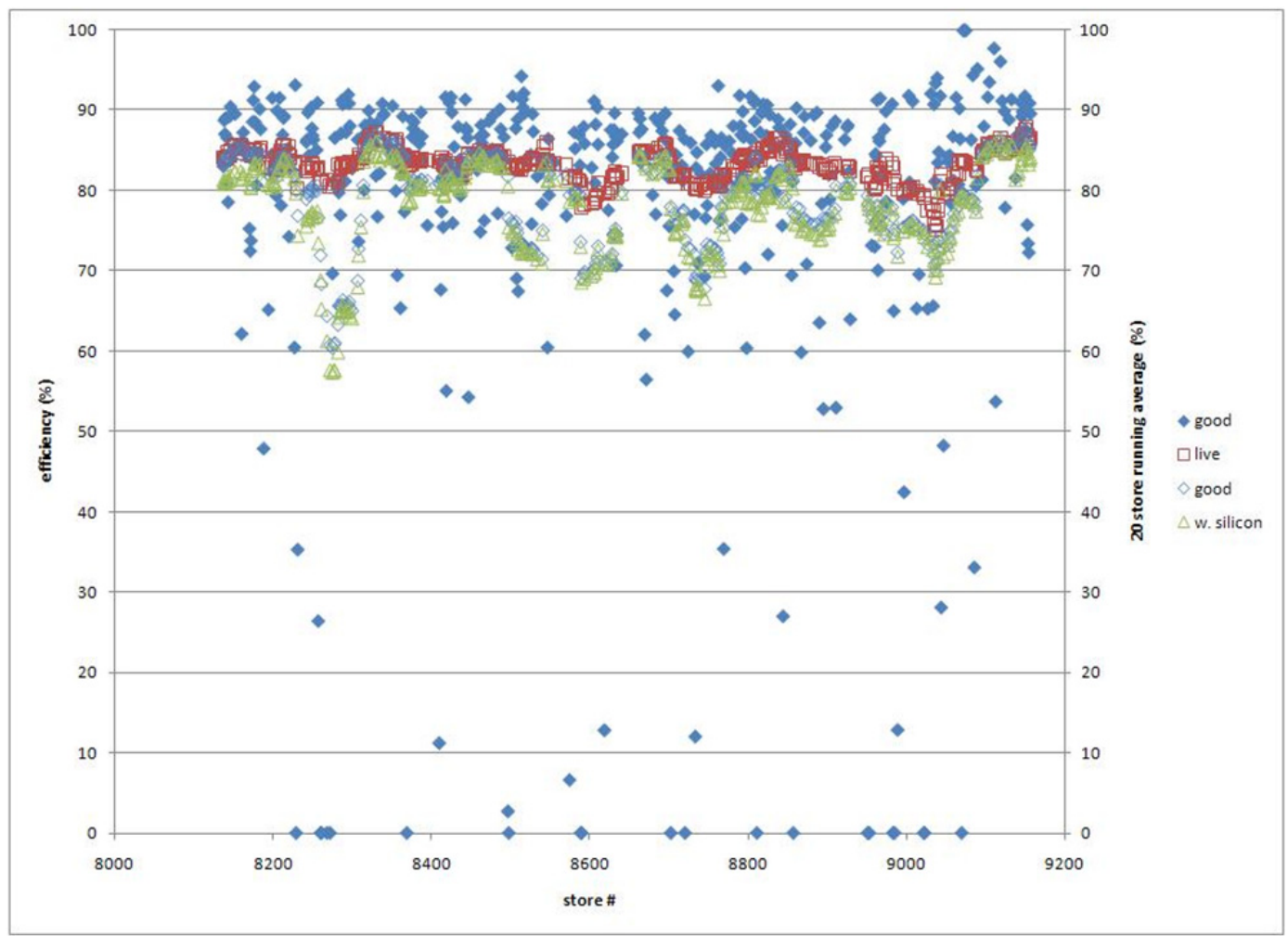

Figure 5. Data-taking efficiency for CDF in fiscal year 2011; blue, store-by-store; hollow symbols 20 store running average for live, good, and full detector (blue, red, green respectively)

During the year CDF integrated diffractive triggers into the default trigger table. Previously CDF had supported requests for diffractive data with dedicated runs. By moving the triggers, CDF was able to fill the bandwidth at low luminosities and acquire more data at essentially zero cost to other analyses. There was also approximately 1 week of "low s" running. Data were acquired at 300 and $900 \mathrm{GeV}$ center-of-mass energies for special analyses.

There were no major changes in shift operations. CDF continued to move responsibilities around as institutions and people gradually left. CDF has moved a few systems per year for the past couple of years; and these adjustments were accomplished again this fiscal year without issue. Towards the end of the year, CDF began planning for final calibrations and securing the detector, Detector Hall and building. On September 30, 2011 the Tevatron was turned off, data taking ceased, and CDF implemented those plans.

\section{$\underline{\text { E-823 / DZero (DZero) }}$ (G. Bernardi, D. Denisov, G. Ginther, S. Gruenendahl, W. M. Lee)}

The accelerator complex delivered an integrated luminosity of $2.56 \mathrm{fb}^{-1}$ to DZero during FY 2011. DZero recorded $2.32 \mathrm{fb}^{-1}$, corresponding to an operating efficiency of $90.5 \%$ during that time period. Figure 6 illustrates the detector efficiency as a function of time during Run II. The FY 2011 performance represents the best yearly performance to date for delivered and recorded luminosity at DZero. These achievements were made possible by increases in instantaneous luminosities delivered by the accelerator complex, improvements which reduced losses during shot setup, and operational enhancements at DZero. 


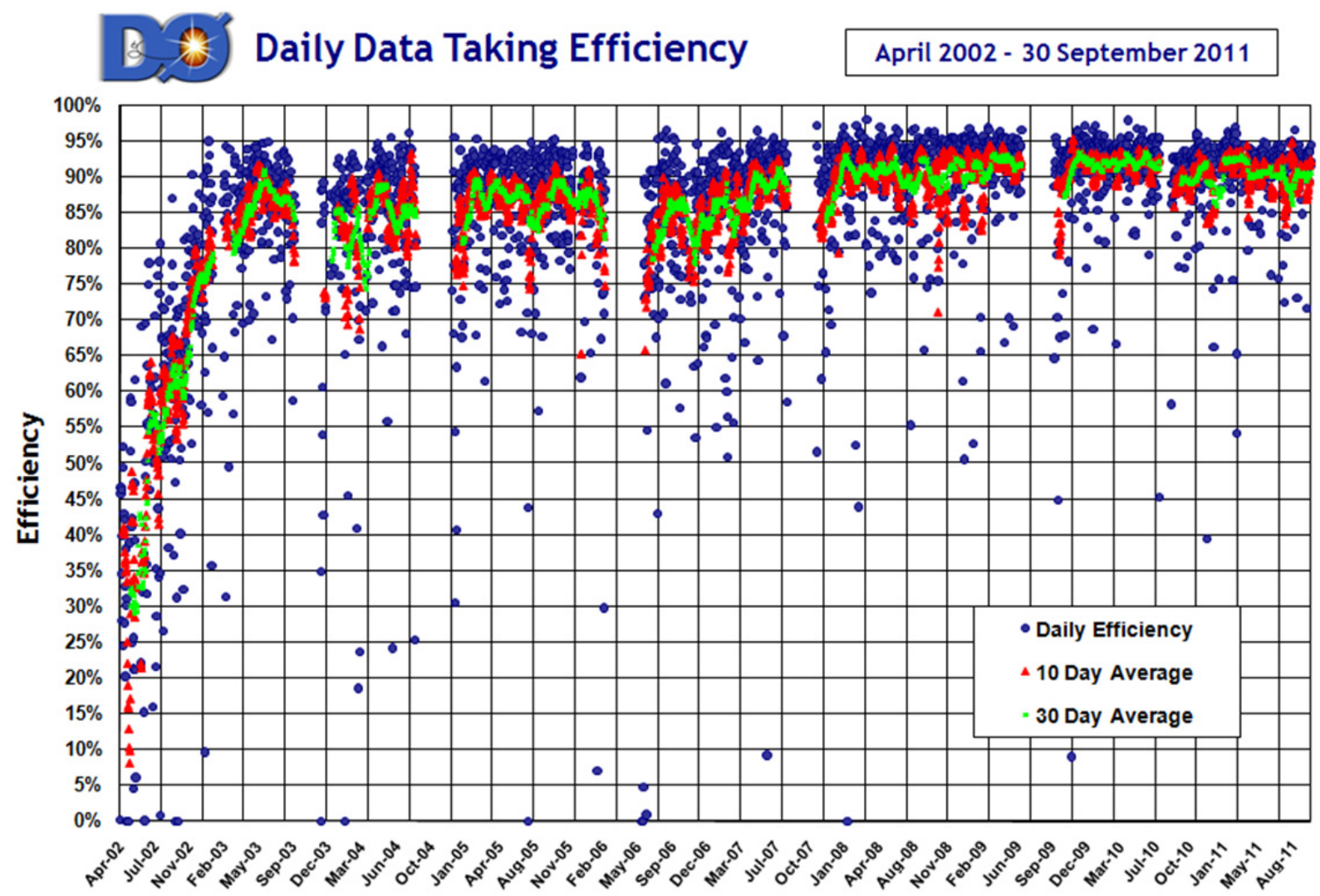

Figure 6. DZero data-taking efficiency as a function of time during Run II. The blue dots represent a daily average, the red triangles represent a 10-day average, and the green squares represent a 30-day average.

DZero continued efforts to streamline operations in the face of increasing instantaneous luminosity, and continued to refine its operations to enhance performance. The operating voltages of the Silicon Microstrip Tracker were adjusted to maintain optimal performance, and monitoring of the impact of radiation damage continued. Hardware and firmware were modified to ensure that the Silicon Microstrip Tracker readout was more fully protected during loss of trigger signals (watchdog implementation).

The Accelerator Division made an adjustment to their scraping procedure in November 2010, which significantly reduced losses during shot setup. Since the Accelerator Division had significantly reduced shot setup losses, DZero eliminated the power down of the muon proportional drift tube readout during shot setup (which was originally introduced to minimize electronics failures).

In early December, the external water supply to the heat exchangers at DZero tripped off, and recovery was complicated by the diversion of water to other areas on the lab site. This infrastructure limitation generated a local dip in operating efficiency in early December.

There was an 11 day interruption in data taking near the end of December due to the failure of a Tevatron component in sector B1. During this downtime, DZero deployed Silicon MIcrostrip Tracker (SMT) readout firmware modifications which provided improved signal to noise, enhanced the SMT calibration procedures, and also suppressed unnecessary run-pausing 
SMT high-voltage alarms. The firmware for the readout of scalers was modified to address a long standing feature (which had not directly impacted data quality, but had the potential to do so if certain conditions were satisfied).

The increasing peak luminosities delivered by the Tevatron generated higher trigger rates and thus larger deadtimes. The record initial luminosity at DZero was $4.2 \times 10^{32} \mathrm{~cm}^{-2} \mathrm{~s}^{-1}$. DZero introduced more frequent prescale transitions and continued to optimize its trigger suites to address these new peak luminosities as well as to mitigate detector aging effects.

Beginning in late February, trigger prescales were adjusted to accumulate a larger fraction of calorimeter calibration data and the resulting small increase in readout deadtime is the principal cause of the $\sim 1 \%$ reduction in average operating efficiency during the final few months of collider operations. The triggers that were routinely used to monitor detector status between stores were also improved to enhance sensitivity to potential calorimeter noise (to provide early warning of possible developing problems).

In March of 2011, there was a scheduled five day downtime. Remotely controlled transfer switches and spare power supplies for sequencers in the SMT readout system were installed during this downtime. This remotely controlled spare power supply capacity was used to allow DZero to take high quality data for two stores that would otherwise have been severely compromised. An improved version of the firmware for the Central Fiber Tracker and Preshower readout boards was also deployed during this shutdown. The Level 2 trigger code was modified to enhance protection against failures during run transitions.

Failures of chillers during June and July resulted in higher than usual humidity levels in the collision hall, and impacted detector performance, briefly compromising muon proportional drift tube coverage.

In addition to ongoing efforts to address remaining sources of dataflow interruptions, there were continuing efforts to streamline performance by improving monitoring tools, and the guidance, training, and documentation provided for the shift crews that operated the detector around the clock.

The average operating efficiency of the detector continued its gentle rise, and was $89.3 \%$ averaged over the entire Run II data sample. As illustrated in Fig. 7, the total integrated luminosity delivered to DZero during Run II was $11.9 \mathrm{fb}^{-1}$, and the total recorded luminosity was $10.7 \mathrm{fb}^{-1}$. 


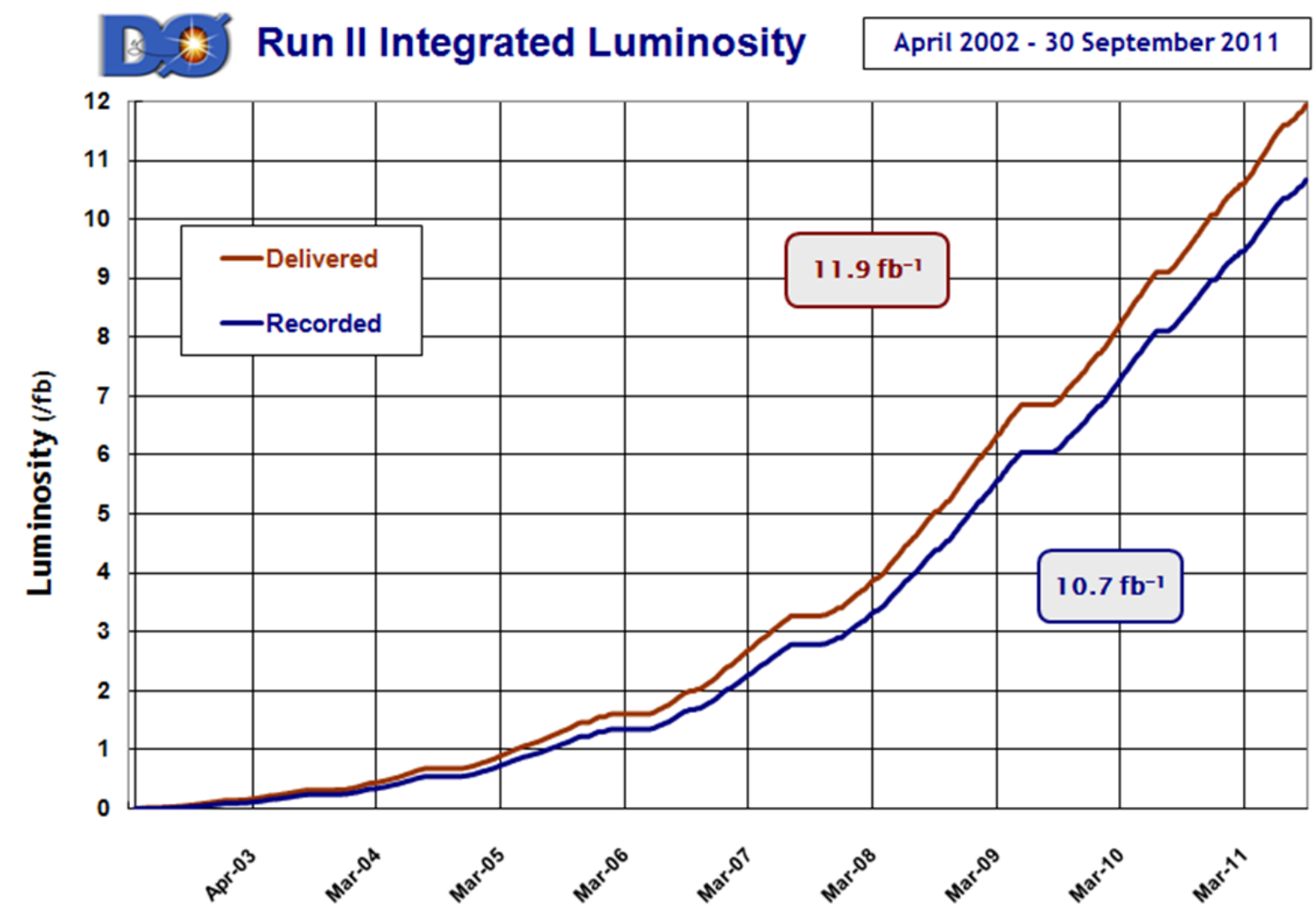

Figure 7. Delivered (red line) and recorded (blue line) luminosity at DZero as a function of time during Run II.

DZero continued to publish refereed-journal papers at an average rate of about three papers a month. The collaboration is well positioned to take full advantage of the substantial accumulated data samples and to continue its multifaceted exploration of particle-physics frontiers.

\section{NuMI Beam (P. Adamson)}

FY 2011 was a challenging year for the NuMI beam. At the start of the fiscal year, NuMI was off for completion of the assembly and installation of NuMI target NT-05. The new target was installed, aligned, and normal beam operations were resumed on November $1^{\text {st }}$, running in low energy anti-neutrino mode. On November $11^{\text {th }}$, the target developed a small leak between the water cooling lines and the helium-filled target can. Running with the helium pressure higher than the pressure in the water lines ensured that this caused a leak of helium into the water system, which is relatively innocuous, rather than a leak of water into the target can, which would cause rapid and complete failure of the target can. 
FY11 NuMI protons

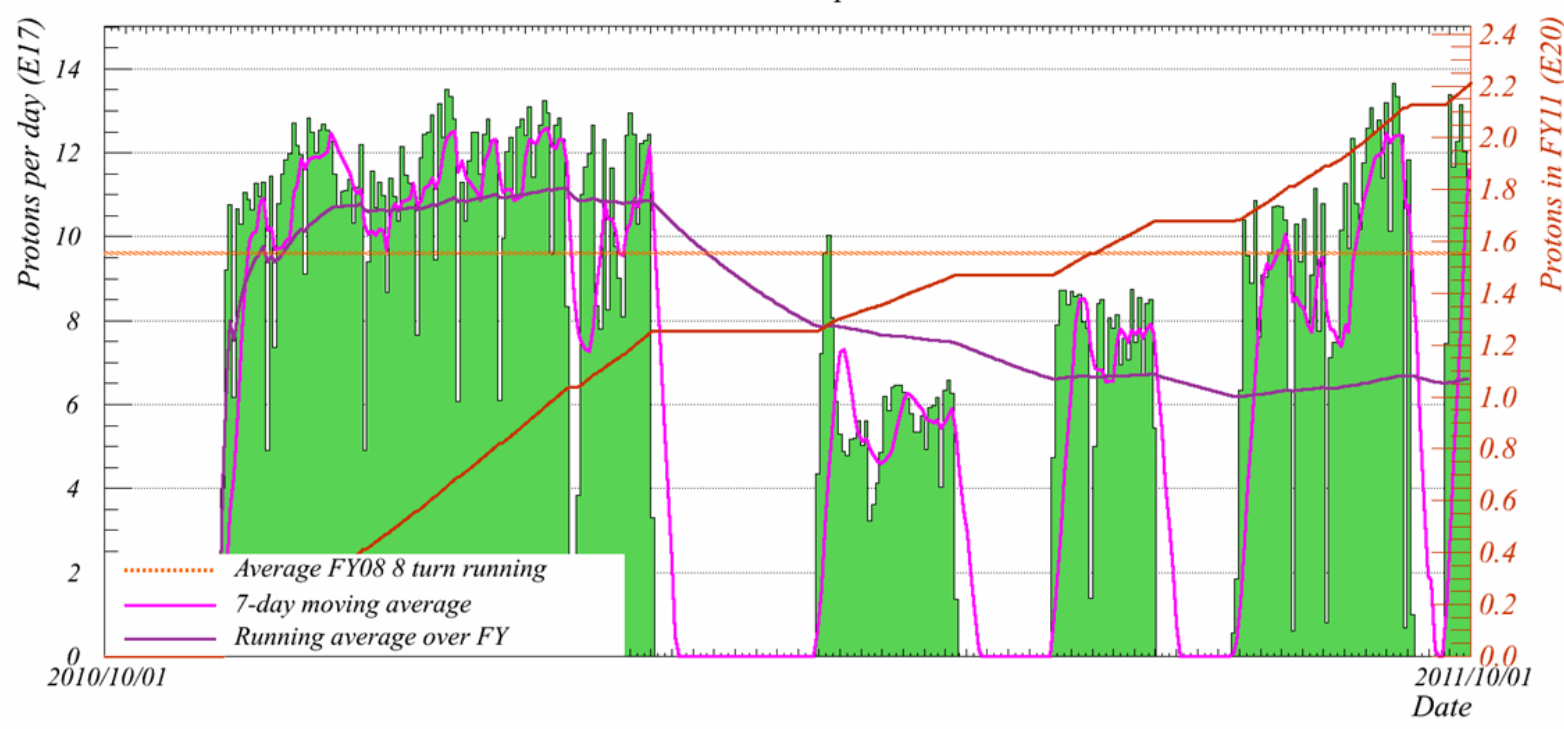

Figure 8. Protons delivered to the target of the NUMI beamline during Fiscal 2011.

The leak was monitored as it became progressively worse over time, until on February $24^{\text {th }}$, the water cooling lines failed completely and the target was removed. An examination of the target revealed that the downstream water turnaround had separated completely from the target cooling water lines, due to failure of a weld.

Target NT-06 was inspected via X-ray, and the weld appeared worse than the one that had failed on NT-05. After the earlier target failures, a program to design a new turnaround was begun, culminating in the decision to remove the suspect turnaround from NT-06 and replace it with a more robust in-house design.

The modified NT-06 was installed, aligned and beam operations resumed on April 9, running in low-energy neutrino mode. On April 12, it too developed a leak between the water lines and the helium can. In the absence of a spare target, the facility was operated at reduced intensity in an attempt to limit the mechanical stresses on the target. The target eventually failed on May $16^{\text {th }}$. A post-mortem examination revealed that the modified water turnaround piece appeared undamaged, but that a significant water leak was present in the upstream portion of the can. This led to modifications to the upstream portions of the water lines on target NT-07 to make them more robust.

Target NT-07 was not due to be ready until the fall, so in the interim, used targets NT-01 and NT-02 were resurrected. NT-01 had a significant external leak which could be repaired, and a small internal leak which could not, and ran for about a month until failure. NT-02 was reinstalled on July $22^{\text {nd }}$, but beam operations were delayed until July $29^{\text {th }}$ by the failure and replacement of the electric cabling for the V108 dipole. NT-02 ran until new target NT-07 was ready in mid-September. NT-02 did not have a water leak, but did have about $15 \%$ reduced neutrino yield in the energy range of interest to the experiments, which was attributed to radiation damage of the graphite target segments. NT- 02 has now been exposed to $6.5 \times 10^{20}$ protons. NT-01 ran in the high and low energy neutrino modes for MINERvA beam-modeling studies. NT-02 ran in low energy antineutrino mode. 
To complete the fiscal year, new target NT-07 was installed and began operating on September $24^{\text {th }}$, in "LE100" medium energy mode for MINERvA beam-modeling studies. This target has both the new Fermilab-design downstream water turnaround piece and redesigned upstream water lines. At the time of writing this report, NT-07 continues to operate without a leak in the water lines.

Table II. FY 2011 NuMI running conditions and proton beam delivered.

\begin{tabular}{|l|c|}
\hline Beam condition & Protons delivered \\
\hline LE010 neutrinos & $0.3 \times 10^{20}$ \\
\hline LE010 antineutrinos & $1.7 \times 10^{20}$ \\
\hline LE100 neutrinos & $7 \times 10^{18}$ \\
\hline LE100 antineutrinos & $1 \times 10^{18}$ \\
\hline LE250 neutrinos & $8 \times 10^{18}$ \\
\hline Tota1 & $2.2 \times 10^{20}$ \\
\hline
\end{tabular}

MINOS Operations (S. Hahn, B. Pahlka, R. Plunkett)

MINOS was not taking data at the start of FY 2011 due to the failure in FY 2010 of target NT-04 and the completion of the carrier assembly and installation of target NT-05 in the LE10 position with the target in the horn. Beam resumed November $1^{\text {st }}$ with the polarity of the horn current reversed in order to produce an antineutrino beam. On February 1, 2011 the horn current was put in the forward direction to provide a systematic study with neutrinos at the same position. What was meant to be a ten day run became much longer due to a variety of problems: problems with near-detector toroidal magnet which eventually required repair work on the magnet, power outages, network problems on the far detector, and, finally, a pair of target leaks starting February $24^{\text {th }}$. The first leak was external and fixed by the next day, but the second leak required a new target, NT-06, which had to be assembled, checked, and installed. Beam resumed April $9^{\text {th }}$ with reduced intensity $\left(1.4 \times 10^{13}\right.$ protons per pulse $)$ due to a helium leak in the target.

Meanwhile, on March $17^{\text {th }}$, a fire at the Soudan mine was acted on quickly by the mine crew who immediately shut down power to the far detector. The following day 50,000 gallons of water and foam were pumped down the shaft. By March $22^{\text {nd }}$ first access was made to the far detector hall. Foam had entered the hall and soaked the magnet coil and uninterruptible power supply, but not the electronics. By April $11^{\text {th }}$, a power cable to replace the damaged cable which ran the length of the shaft had arrived. By the following week, power was restored to the far detector hall and elsewhere, and new fiber optic cables were installed. By April $26^{\text {th }}$, the far detector data-acquisition system was up and running on cosmic rays. By May $9^{\text {th }}$, the damaged sprinkler system was replaced, and magnet coil testing started. The magnet was returned to normal operating levels May 19 after a drying out period at lower voltages.

On June $11^{\text {th }}$, beam returned to both the near and far detectors running with full magnetic fields after the NT-01 target was installed in the LE250 position and beam was run at $2.5 \times 10^{13}$ protons per pulse. On June $22^{\text {nd }}$, the target was moved to the LE10 low-energy beam position and run for until July 8 with the intensity slowly being raised to $3 \times 10^{13}$ protons per pulse. 


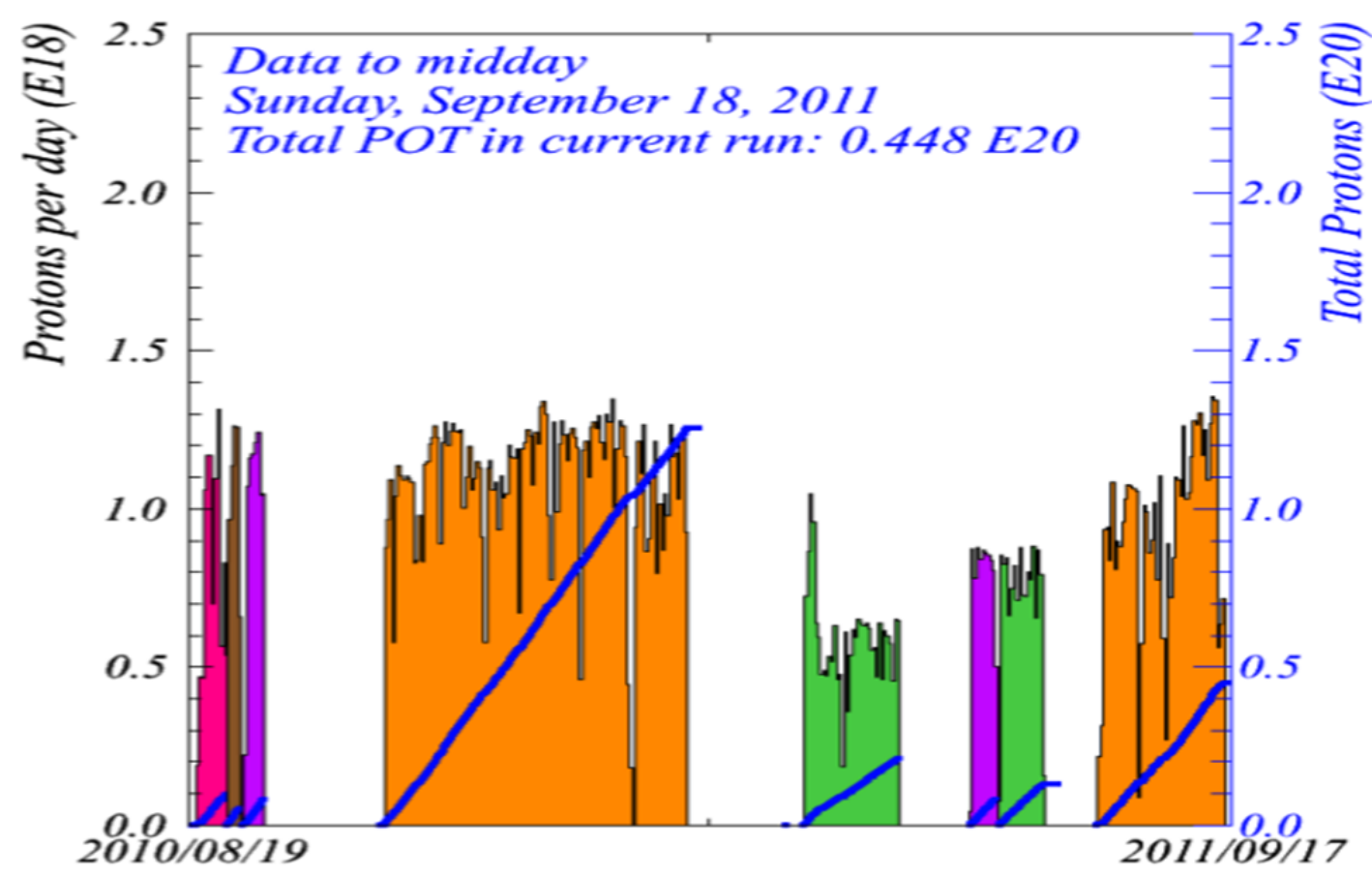

Figure 9. Overview of protons delivered to the target of the NUMI beamline during Fiscal 2011.

A variety of tasks were performed by MINOS during the short summer shutdown:

- Target NT-01, which had a helium leak problem, was replaced with the reusable target NT-02.

- Fan packs continued to be repaired throughout the shutdown by CDF technicians. This work had started before the shutdown started.

- Repairs were made to the air handlers in response to the increasing hall and electronics temperatures.

- Investigations of continuing problems in a readout-processor crate were done over the course of the shutdown. An interface card in the branch PC and a readout processor board were replaced.

- A new test crate upstairs in the MINOS building was assembled during the shutdown and afterwards to allow independent investigations without incurring downtime for the near detector.

Beam returned to NuMI on July $29^{\text {th }}$ with intensity slowly ramping from about $3.0 \times 10^{13}$ protons per pulse for most of August up to the nominal $3.5 \times 10^{13}$ protons per pulse the second week of September. The horn current was reversed (anti-neutrino mode). Running proceeded smoothly through the remainder of the fiscal year. 
MINOS continued in FY 2011 to publish results on the fundamental parameters of the oscillations of muon neutrinos. The precise data set is currently based on analysis of $7.25 \times 10^{20}$ POT and has been published as PRL 106181801 (2011). The results of this analysis are the world's most precise for the mass-squared difference and are displayed below in Fig. 10.

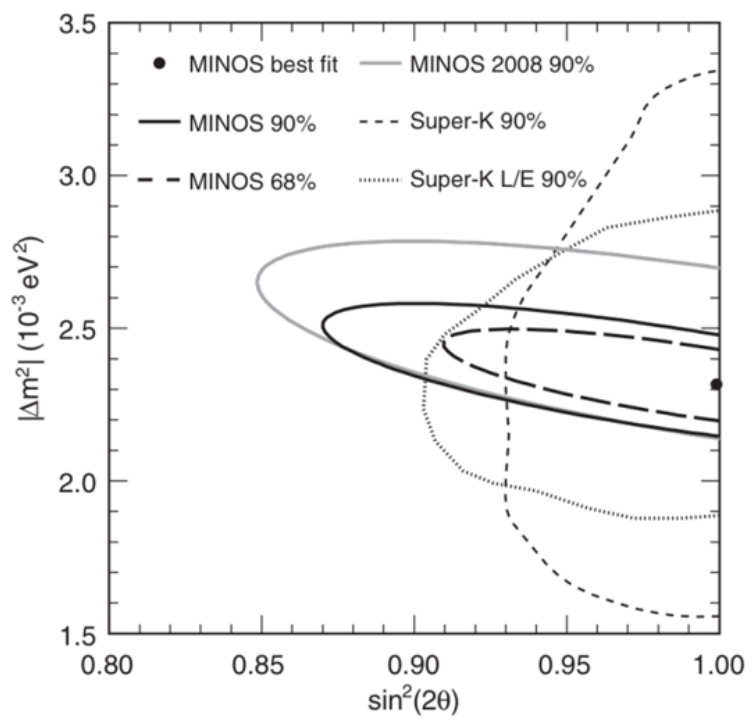

Figure $10.68 \%$ and $90 \%$ confidence limits around MINOS best fit values for the oscillation parameters of muon neutrinos.

In FY 2011 MINOS published earlier results on the oscillation parameters of muon antineutrinos and presented preliminary results on a larger dataset of antineutrinos. The earlier results had shown some tension with the precisely measured parameters of muon neutrino oscillations. The larger dataset analyzed and presented in FY 2011 gave parameters much more similar between neutrinos and antineutrinos, giving weight to the hypothesis that the earlier result was due to statistical fluctuation. The result is shown in Fig. 11, and is in preparation for publication. 


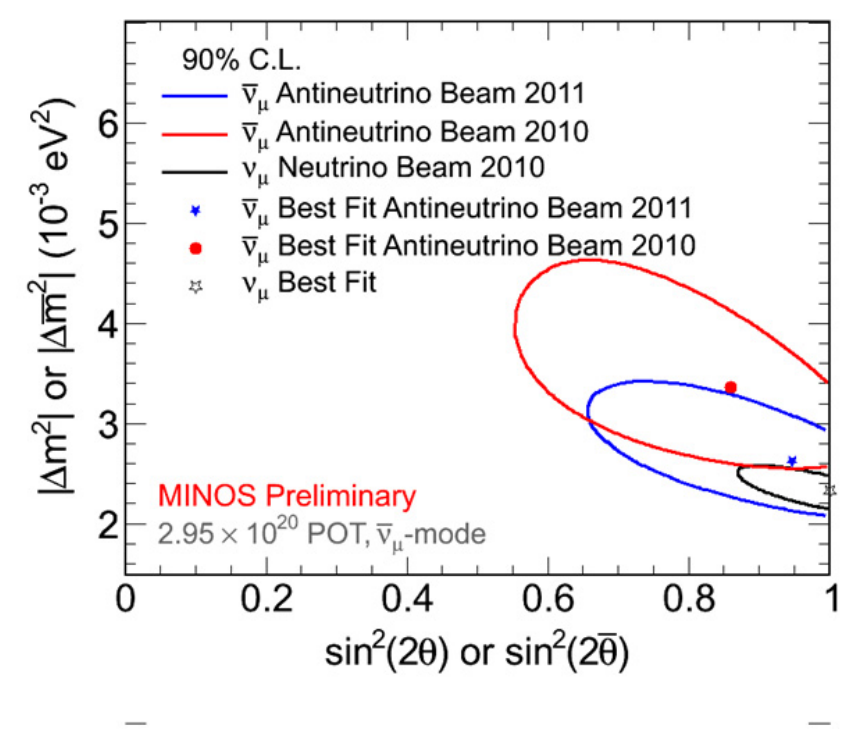

Figure 11. Combined plot of MINOS measured values for the oscillation parameters of neutrinos and antineutrinos. Best fit values and $90 \%$ contours are shown in black and blue, respectively. For comparison, the results of an earlier measurement are shown in red.

MINOS can also search for the appearance of electron neutrinos in the muon neutrino beam. This continued to be a subject of great interest during FY 2011. MINOS extended its work in this area in two ways: addition of more data, and maximizing the sensitivity of the analysis technique. The result shown in Fig. 12 is based on observation of 62 electron-like events over a predicted background of 50 . The case of zero appearance is ruled out at $89 \%$ significance.

In addition to oscillation experiments, MINOS published results in FY 2011 concerning limits on active-to-sterile neutrino mixing (using neutral currents) and topics concerning cosmic rays. Of particular interest, a publication placing strong bounds on Lorentz violating models which create sidereal variations in neutrino interaction rate also appeared. 


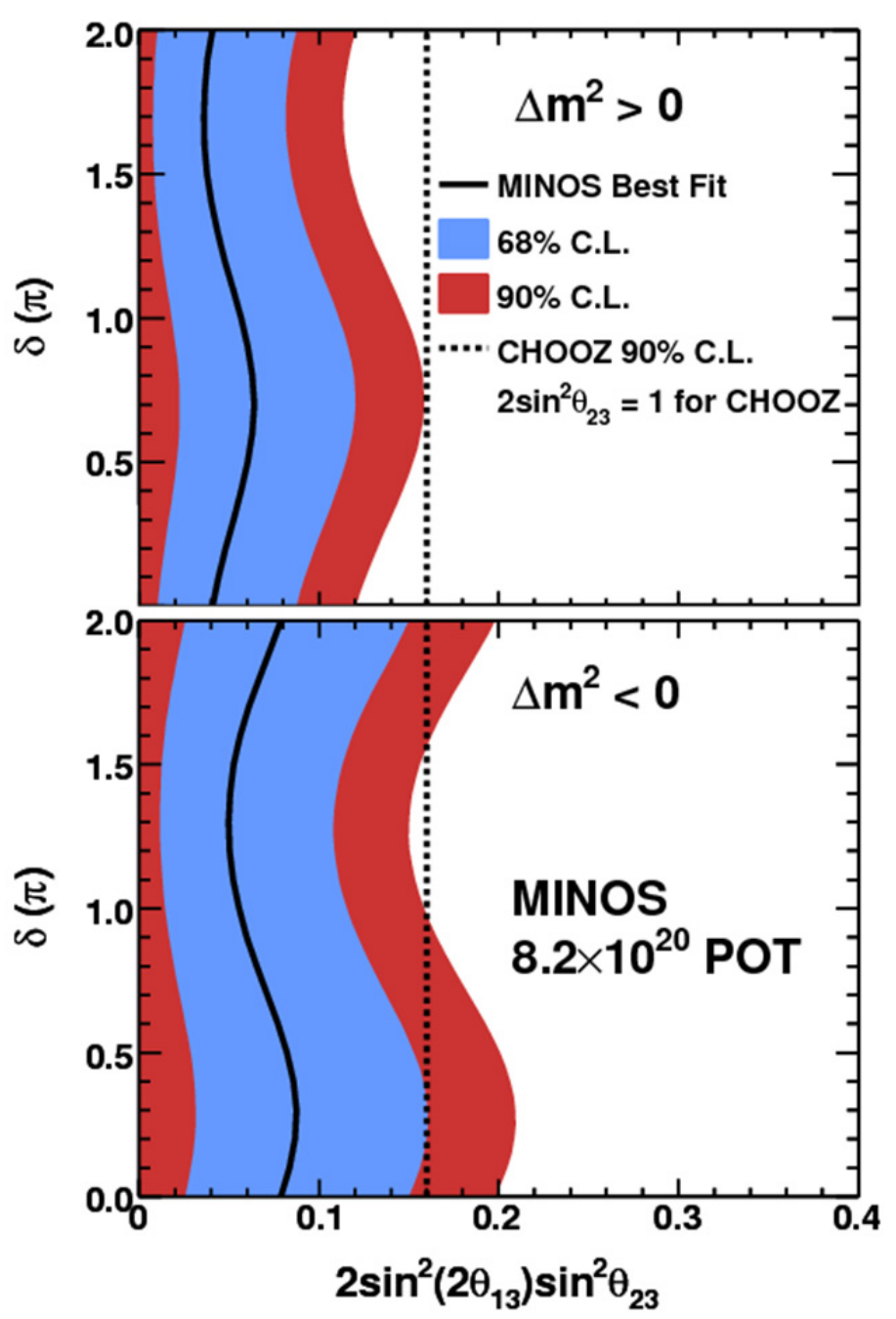

Figure 12. Limits and best fit values obtained by the MINOS experiment for electron-neutrino appearance using the NuMI beam. The top (bottom) figure shows the limit compared to expectations for the normal (inverted) mass hierarchy.

E-938 / MINERvA (D. Harris, K. McFarland)

\section{MINER vA Construction and Installation Activities in FY 2011}

The MINERvA experiment began taking data on the partially completed detector in November 2009 while the last components were being built and commissioned. By mid-March 2010 the installation was completed and the experiment switched over to a new data acquisition system and started taking data with the full active detector, and all of the solid targets installed.

As part of an NSF MRI grant the MINERvA experiment designed and procured a cryogenic target which was delivered to Fermilab on January 11, 2011. The target and the cryocooler were extensively tested above ground before installation underground, which took place on March 23, 2011. Additional work underground was required for the filling, ventilation, and monitoring systems, and by early summer the system was ready for safety review. An additional relief valve was installed over the summer and the vessel was filled with liquid helium in time for the return of the low energy neutrino beam that occurred in the beginning of October 2011. 
In order to ensure that events that look like they originate from neutrino interactions in the helium are not in fact due to the far more copious muons produced in upstream neutrino interactions, a veto wall was assembled and commissioned in FY 2011. This "wall" consists of two planes of scintillator panels extending roughly 15 ' tall by 10 ' wide, placed downstream of a 1 " and 2" thick plane of steel, respectively. This wall had to be assembled and commissioned in the beamline after the cryogenic target was installed. The work occurred in August 2011 when it was clear that the helium target was going to pass safety reviews without substantial additional work at the upstream region of the target. The veto wall was commissioned in September 2011 and was operational by the return of the low energy neutrino run in early October 2011.

As part of the same NSF MRI grant, in FY 2011 the experiment, with the help of the Fermilab PPD, also completed the design and fabrication of a new Kevlar and steel-based water target. This target uses two steel rings and a spacer ring, and Kevlar and vapor barriers are stretched between the rings. Water is added through a penetration in the spacer ring. The rings were delivered on July 27, 2011, and commissioning of the target (and construction of the water target stand) proceeded through the end of the fiscal year. The water target itself has to fit within a 10 1/2" gap in the MINERvA detector stack, and between July and October 2011, test fills were completed and the rings were reinforced well enough so that in early FY2012 the water target was successfully installed underground. The acrylic water target that was described in an earlier report was removed from the detector stack once it was clear the new water target would present a viable alternative. Graphical representations of recorded events with neutrino interactions in the water and helium targets are shown in Fig. 13.

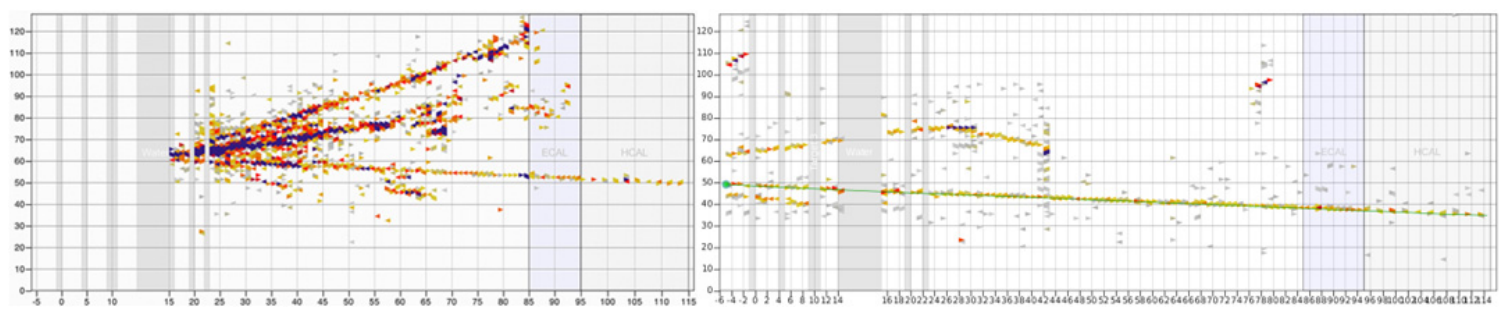

Figure 13. Left: a candidate neutrino interaction that originates in the (passive) water target. Right: a candidate neutrino interaction that originates in the upstream cryogenic target (not shown) that is filled with helium. The lack of activity in the upstream veto region of the detector is also not shown. For both displays, the color in each pixel is a function of the energy deposited in that pixel.

Because the MINERvA data analyses depend critically on the MINOS near detector being operational, MINERvA personnel involvement ramped up in FY 2011 so that MINERvA collaborators are part of the detector expert crew that addresses MINOS near detector electronics and DAQ problems, along with MINOS and PPD personnel.

A final operational upgrade that occurred over FY 2011 is the capability and the subsequent approval to staff remote shifts for the MINERvA experiment. The necessary hardware infrastructure was developed in early 2011 at the University of Rochester, duplicated and sent to several remote institutions based on interest and need, and by April 2011 the University of Rochester had completed the necessary tests to be certified as a remote shift location. By the end of FY2011 there were four remote sites qualified: University of Rochester, Tufts University, University of Texas at Austin, and the University of Santa Maria in Valparaiso, Chile. By the end of FY2011 the experiment was running approximately one third of its shifts remotely. 


\section{Physics Goals for MINER vA in Low Energy Beam}

The goal during the low-energy neutrino running is to provide exclusive cross-section measurements on a variety of nuclei. The data will help in understanding the details of neutrino interactions, and serve as important constraints for current and future neutrino oscillation experiments. Low energy events tend to have few final state particles - which allows the MINERvA detector to identify single particles and the exclusive channels important for current and future oscillation experiments. MINERvA's detector is about a factor of 10 more finegrained than the NOvA detector, and can identify things that contribute backgrounds to NOvA. MINERvA sees a higher neutrino beam energy than T2K's near detector, and measures reactions that contribute backgrounds to T2K from the high-energy tail of the beam (where "high energy" in the T2K case means above $1 \mathrm{GeV}$ ). Low energy data will also allow a study of exclusive channels as a function of target nucleus. Various processes are expected to have different nuclear dependencies. MINERvA should be able to see those different dependencies clearly if it accumulates the expected amount of data.

\section{Time Line of MINER vA Operations}

MINERvA started taking physics-quality data with its partially completed detector in November 2009. For this detector, the entire downstream hadron and electromagnetic calorimeters were installed, as well as about half the active target modules. Between November and January there was also one nuclear target containing both iron and lead, and a veto region before the active tracker volume, allowing first nuclear target studies with antineutrinos. After January the nuclear target and veto region were removed to continue installation of the remaining $45 \%$ of the detector. During the second installation period, from January 2010 through March 2010, the downstream detector components remained in a stable configuration and the experiment continued to take antineutrino data.

Between the start of the MINERvA anti-neutrino run until March 1, 2010, the ArgoNeuT Liquid Argon TPC test was located between the MINERvA detector and the MINOS near detector. This represented about 2 metric tons of passive material in a complicated geometry that intersect approximately $40 \%$ of the muons that start from the fiducial region of MINERvA and pass into the MINOS near detector. The average energy loss passing through ArgoNeuT was about $100 \mathrm{MeV}$, but the variation of the energy loss is large from event to event, and in some regions up to a $\mathrm{GeV}$ of energy would be lost between MINOS and MINERvA. ArgoNeuT was removed from between the two detectors on March 1, 2010. During FY2011 MINERvA developed the necessary detailed Geant-based simulation to correctly simulate on an event-byevent basis the energy loss that will occur for the muons crossing through the region.

On March 22, 2010 the detector installation and checkout was completed and the neutrino running began. This run includes all of the solid nuclear targets that are planned for the experiment. The livetimes quickly reached the $95 \%$ level and above after about a week of running, and have been stable and high since that time. For FY 2011 alone the integrated livetime of the MINERvA detector was $98.1 \%$, collecting $2.16 \times 10^{20}$ protons on target (POT) of the $2.20 \times 10^{20}$ POT delivered in FY 2011 . 
The data taken in FY 2011 span a few different periods: from November 2010 through April 2011 the experiment recorded approximately $1.47 \times 10^{20}$ POT in anti-neutrino mode, followed by a shutdown to change targets, then a brief run in neutrino mode June 11 through July 7 , including special runs to better understand the neutrino flux. Unfortunately production target problems developed and because a new target was not yet ready, starting July 29 the experiment recorded another $0.44 \times 10^{20}$ POT in anti-neutrino data with an older target (NT-02) that was reinstalled, but which had much larger flux uncertainties than other targets due to the large exposure it had received prior to MINERvA running. By September 24 a new target (NT-07) was installed and the experiment resumed data taking, starting with three special runs to get closer to the original special run request. The total number of POT for neutrino running in FY 2011 was therefore $0.47 \times 10^{20} \mathrm{POT}$, roughly $10 \%$ of the total request of $4.9 \times 10^{20}$ POT over what was one half of our expected pre-NOvA run period. The low-energy neutrino running resumed by October 6, 2011. The number of integrated protons on target in each running mode is shown in Table III.

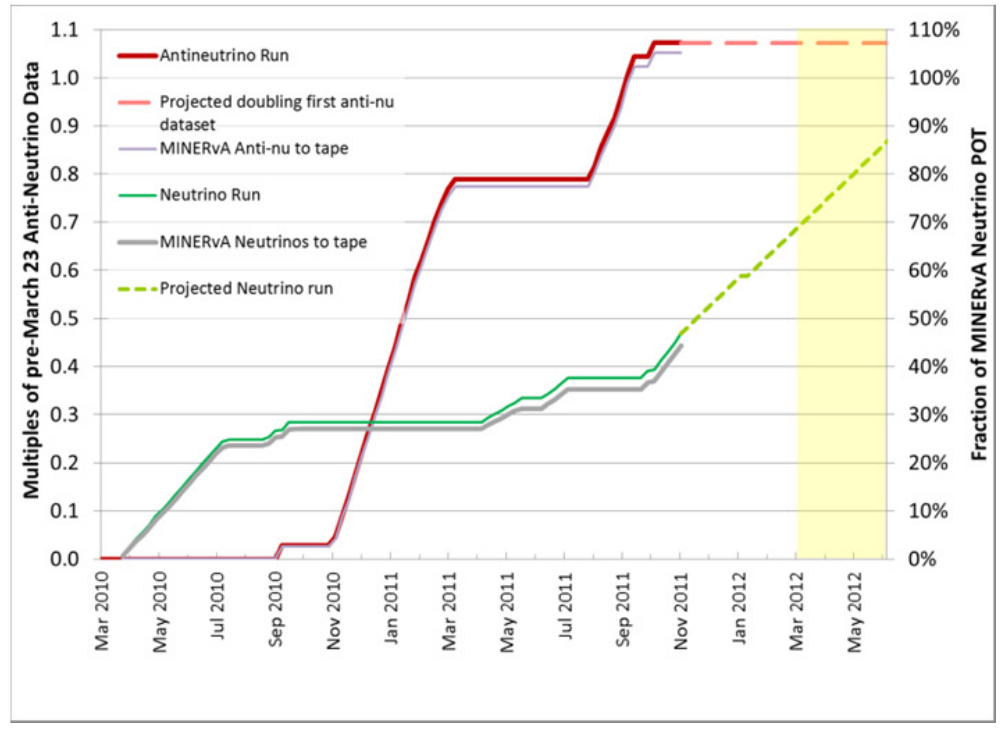

Figure 14. Recent NuMI running, the MINERvA data written to tape, and one possible projected future run. The projection assumes the NuMI complex will repeat its past performance over a one-year uptime average without any future long downtime except for one two-week shutdown for a target change. The red line shows the run that doubled the MINOS pre-March 2010 anti-neutrino exposure. The green line shows a run following the anti-neutrino run to give MINERvA its full requested low-energy neutrino exposure. The shaded yellow area is time after the current date for the shutdown to begin NOvA improvements to the Main Injector and to the NuMI beamline. 
Table III. Normal and special runs for the low-energy-target running, as well as the protons recorded in each configuration in FY 2010 and FY 2011.

\begin{tabular}{|l|l|l|l|}
\hline Run & Dates & POT Recorded & Goal \\
\hline $\begin{array}{l}\text { Target at 100cm, Forward } \\
\text { Horn Current 200kAmps }\end{array}$ & $\begin{array}{l}8 / 26 / 10-9 / 3 / 10 \text { and } \\
9 / 24 / 11-9 / 29 / 11\end{array}$ & $\begin{array}{l}7.0 \times 10^{18} \\
7.0 \times 10^{18}\end{array}$ & $15 \times 10^{18}$ \\
\hline $\begin{array}{l}\text { Target at 100cm, Reverse Horn } \\
\text { Current 200kAmps }\end{array}$ & $\begin{array}{l}9 / 3 / 10-9 / 8 / 10 \text { and } \\
9 / 30 / 11-10 / 4 / 11\end{array}$ & $5.5 \times 10^{18}$ & and \\
\hline $\begin{array}{l}\text { Target at 150cm, Forward } \\
\text { Horn Current 200kAmps }\end{array}$ & - & 0 & 0 \\
\hline $\begin{array}{l}\text { Target at 250cm, Forward } \\
\text { Horn Current 200kAmps }\end{array}$ & $\begin{array}{l}9 / 8 / 10-9 / 17 / 10 \text { and } \\
6 / 11 / 11-6 / 20 / 11\end{array}$ & $\begin{array}{l}7.7 \times 10^{18} \\
8.1 \times 10^{18}\end{array}$ & $15 \times 10^{18}$ \\
\hline $\begin{array}{l}\text { Target at 10cm, Forward Horn } \\
\text { Current 200kAmps }\end{array}$ & - & 0 & $15 \times 10^{18}$ \\
\hline $\begin{array}{l}\text { Target at 10cm, Forward Horn } \\
\text { Current 150kAmps }\end{array}$ & - & 0 & $15 \times 10^{18}$ \\
\hline $\begin{array}{l}\text { Target at 10cm, 0kAmps, } \\
\text { MINOS coil focusing negative } \\
\text { muons }\end{array}$ & $4 / 14 / 11-4 / 21 / 11$ & $7.2 \times 10^{18}$ & $15 \times 10^{18}$ \\
\hline $\begin{array}{l}\text { Target at 10cm, Forward Horn } \\
\text { Current, 180kAmps (nominal } \\
\text { low-energy running) }\end{array}$ & $3 / 21 / 10$ through $10 / 1 / 11$ & $147 \times 10^{18}$ & $400 \times 10^{18}$ \\
\hline
\end{tabular}

\section{Analysis Summary of Test Beam Experiment (T-977)}

The MINERvA test beam detector took data between June 7 and July 16, 2010 in two different configurations. Initially, the upstream 20 planes were configured like the electromagnetic calorimeter (ECAL) of MINER $v$ A (with $2 \mathrm{~mm}$ of lead between each plane), and the downstream 20 planes were configured like the hadron calorimeter of MINERvA (with 1" of steel between each plane). Next, data were taken with no absorber between the first 20 planes of scintillator, and $2 \mathrm{~mm}$ of lead between the downstream 20 planes to model the tracker-ECAL interface of the neutrino detector.

In FY 2011 the test beam analysis efforts were focused on improving the upstream beamline reconstruction (tracking and timing, for example), as well as on incorporating the same detector calibration steps that were already established for the neutrino detector analyses. A new plot of the reconstructed track momentum versus time of flight is shown in Fig. 15 (Left). The different populations of positive and negative particles are clearly seen, as are the accidental triggers that arrive at the next rf bucket 20 nanoseconds later. Fig. 15 (Right) shows the reconstructed masses of the particles in the test beam. 

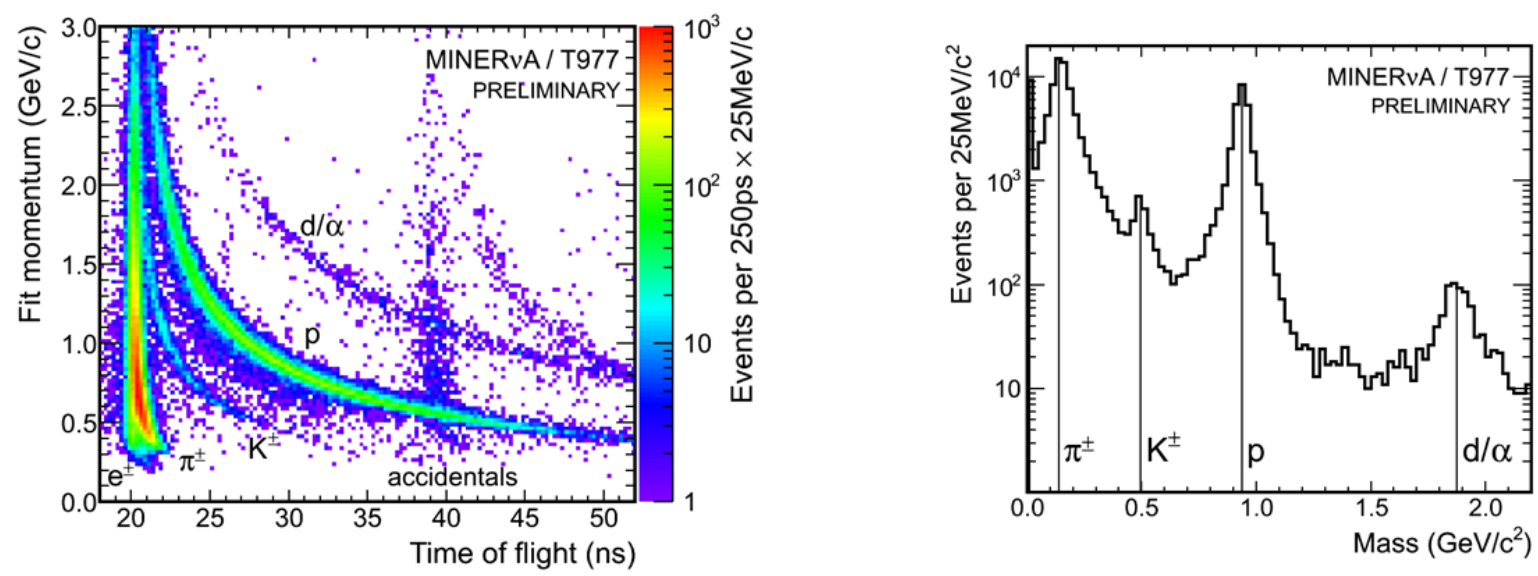

Figure 15. Left: Reconstructed particle momentum versus the time of flight according to the upstream beamline instrumentation in MTest. The different populations of test beam particles are clearly indicated. Right resulting mass spectrum for all triggers taken in the 2010 summer run.

\section{Physics Highlights in MINERvA in FY 2011}

In FY 2011 MINERvA presented its first physics distributions to the public, starting with NuINT11 in Dehradun, India in March 2011, and continuing at the Fermilab User's Meeting in June 2011 and subsequent summer conferences. The two analyses highlighted here show the experiment's progress in Quasi-Elastic (QE) scattering, perhaps the most important neutrino interaction channel for neutrino oscillation experiments, and also the progress in analyzing the special runs in alternate beamline geometries that were taken to better constrain the MINERvA neutrino flux model.

The QE analysis presented at NuINT11 uses the earliest data that MINERvA took, which was when the NuMI beamline was providing anti-neutrinos for the MINOS experiment and only the downstream region of the detector was commissioned. The anti-neutrino Quasi-Elastic signature is particularly straightforward: because the final state particles are a neutron and a muon, the analysis need only identify a positively charged muon and the absence of measured energy more than $5 \mathrm{~cm}$ from the muon track. Neutrons in the final state may deposit some energy away from the muon but the cut described below takes this into account. Quasi-elastic events are identified simply by the fiducial cuts on the interaction vertex, the requirement of a positively charged muon as tracked by the MINOS detector, and the absence of any extra recoil energy that is away from the muon. The recoil is dependent on the momentum transferred in the event, so a $\mathrm{Q}^{2}$ dependent cut was made to reduce the non-quasi-elastic background without removing possible signal events. Plots of both the $\mathrm{Q}^{2}$ and extra recoil energy distributions are shown in Fig 16. 

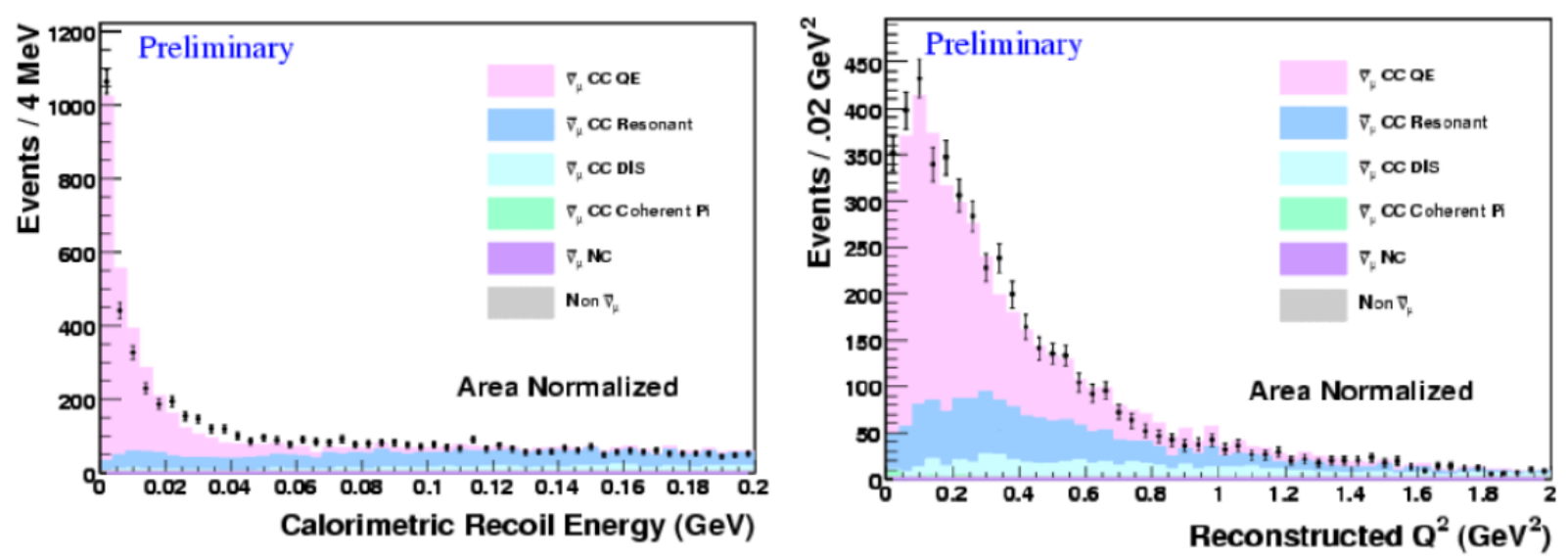

Figure 16. The calorimetric recoil energy away from the muon that is located in the charged current event candidates (left) and the reconstructed Q2 distribution of the remaining events after a cut on calorimetric energy is made (right). Both plots are area normalized and only the statistical uncertainties are shown.

In order to turn the kinematic distribution shown in the above plots into an absolute cross section measurement, the experiment requires knowledge of the absolute neutrino flux that is incident on the detector. The NuMI beamline is unique among neutrino beams in that it can be tuned to provide fluxes of different neutrino energies. Possible modifications either change the relative position between the target and the focusing horns, or change the current in the horns. Taking data in different beam configurations allows the study of different regions of the parent pion kinematics. Hadron production models can then be constrained to match the observed distributions. These measurements may test the model using different neutrino interactions, for example, by using both quasi-elastic and inclusive event rates. As of the EPS 2011 conference, the experiment had taken data in a total of three different target positions, and two different horn currents for the nominal target position data. The neutrino energy distribution for inclusive charged current events in some of the special runs taken is shown in Fig. 17, as is the momentum-transfer-squared distribution in the antineutrino special run data set. In both plots, the data sample shown is only a fraction of the total numbers of events recorded, but show progress in kinematic reconstruction.
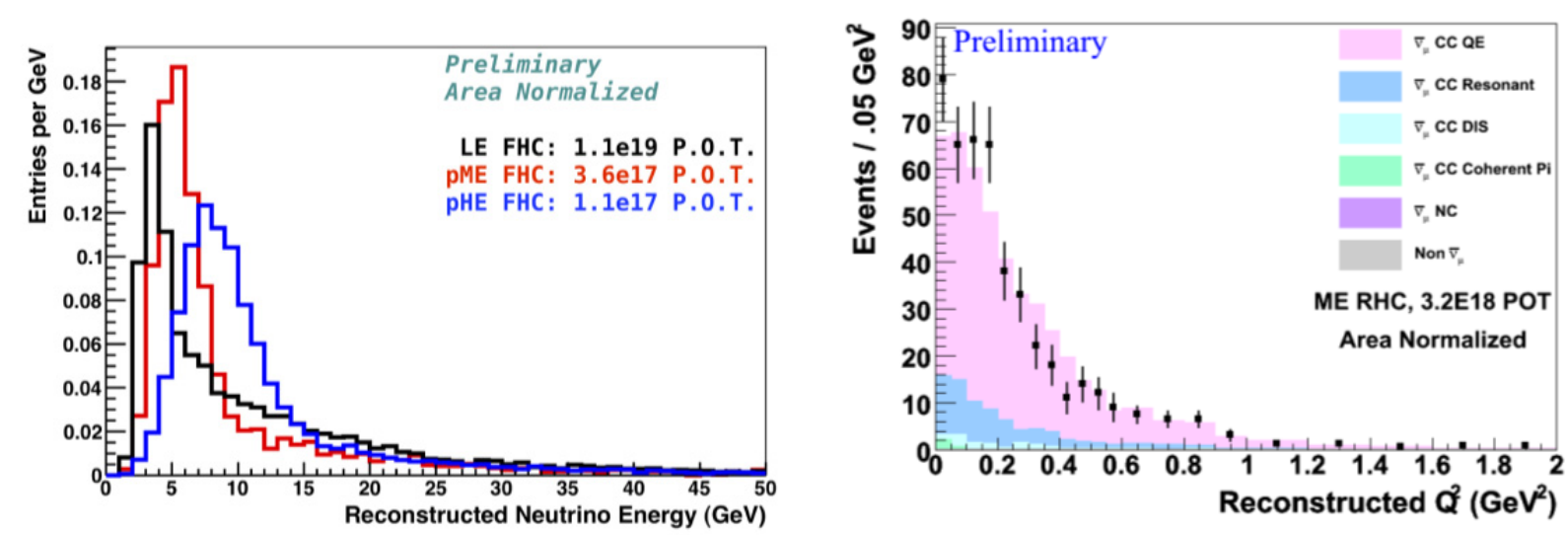

Figure 17. Left: The reconstructed neutrino energy for an inclusive sample of neutrino events in the neutrino mode special run data. The events shown represent a small fraction of data taken, as indicated by the key describing the numbers of protons on target. Right: The reconstructed momentum transferred in both data and simulation for some of the antineutrino special run (medium energy) data set. 


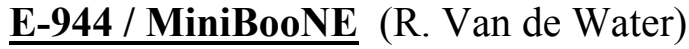

The FY 2011 Booster Neutrino Beam running for MiniBooNE was entirely in antineutrino mode. During this period, MiniBooNE collected data from $3.50 \times 10^{20}$ protons on target (POT). Added to previous antineutrino running, this brought MiniBooNE to a total of $1.01 \times 10^{21}$ POT in antineutrino mode. Antineutrino oscillation results with $5.66 \times 10^{20}$ were published in Phys. Rev. Lett. 105:181801 (2010) and an update with $8.58 \times 10^{20}$ POT was made public this past summer (but not published). Fig. 18 shows closed contours around the LSND preferred region for the $5.66 \times 10^{20}$ (left) and $8.58 \times 10^{20}$ (right) POT, suggesting that in antineutrino mode, MiniBooNE data are consistent with the earlier LSND results. As can be seen, with the extra data, the agreement between MiniBooNE and the LSND two-neutrinooscillations region is reduced. The LSND region, which was previously covered by the MiniBooNE 99\% CL contours, is now only covered by the $90 \%$ CL contours. Clearly more data is crucial to minimize possible statistical fluctuations and ultimately confirm or refute these results. MiniBooNE has been approved for up to $1.5 \times 10^{21}$ protons on target assuming run conditions allow it.

Figure 19 shows the integrated performance of the Booster Neutrino Beam up to the end of FY 2011. The performance of the Booster during FY 2011 was steady, with beam uptime of $85 \%$ and average weekly delivered protons of $0.35 \times 10^{19}$ protons on target/week. The weekly number of protons on target climbed steadily at the end of FY 2011 due to improved Booster performance and periods of NuMI downtime for target replacement. MiniBooNE can take advantage of such unscheduled peak protons-on-target periods due to its high reliability and uptime. Also shown are various projections to the start of the 2012 long shutdown for three proton/hour delivery rates. The actual rate is about $3.0 \times 10^{16}$ protons/hour, and with the $\mathrm{TeV}$ shutdown at the end of FY 2011, this will go up to as much as $3.5 \times 10^{16}$ protons/hour. At this rate, MiniBooNE should get to about $1.20 \times 10^{21}$ by the scheduled 2012 shutdown, which is close to our analysis goal of $1.5 \times 10^{21}$ POT.
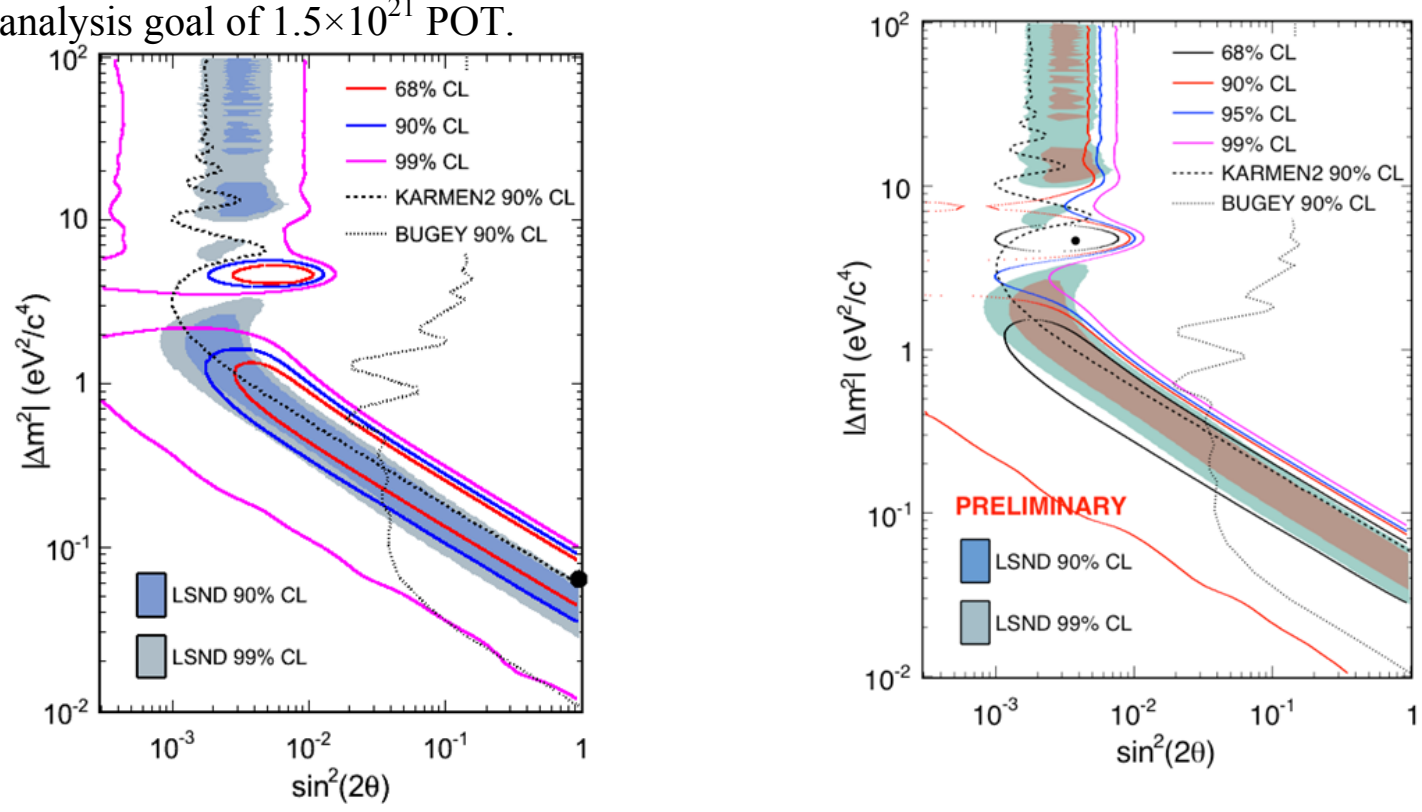

Figure 18. MiniBooNE $68 \%, 90 \%$, and $99 \%$ C.L. allowed regions within a two neutrino oscillation model from $5.66 \times 10^{20} \mathrm{POT}$ (left) and $8.58 \times 10^{20} \mathrm{POT}$ (right) for events with $\mathrm{E}_{\mathrm{QE}}>475 \mathrm{MeV}$. Also shown are limits from KARMEN and Bugey. The shaded areas show the $90 \%$ and $99 \%$ C.L. LSND allowed regions. The black dot shows the best fit point. 


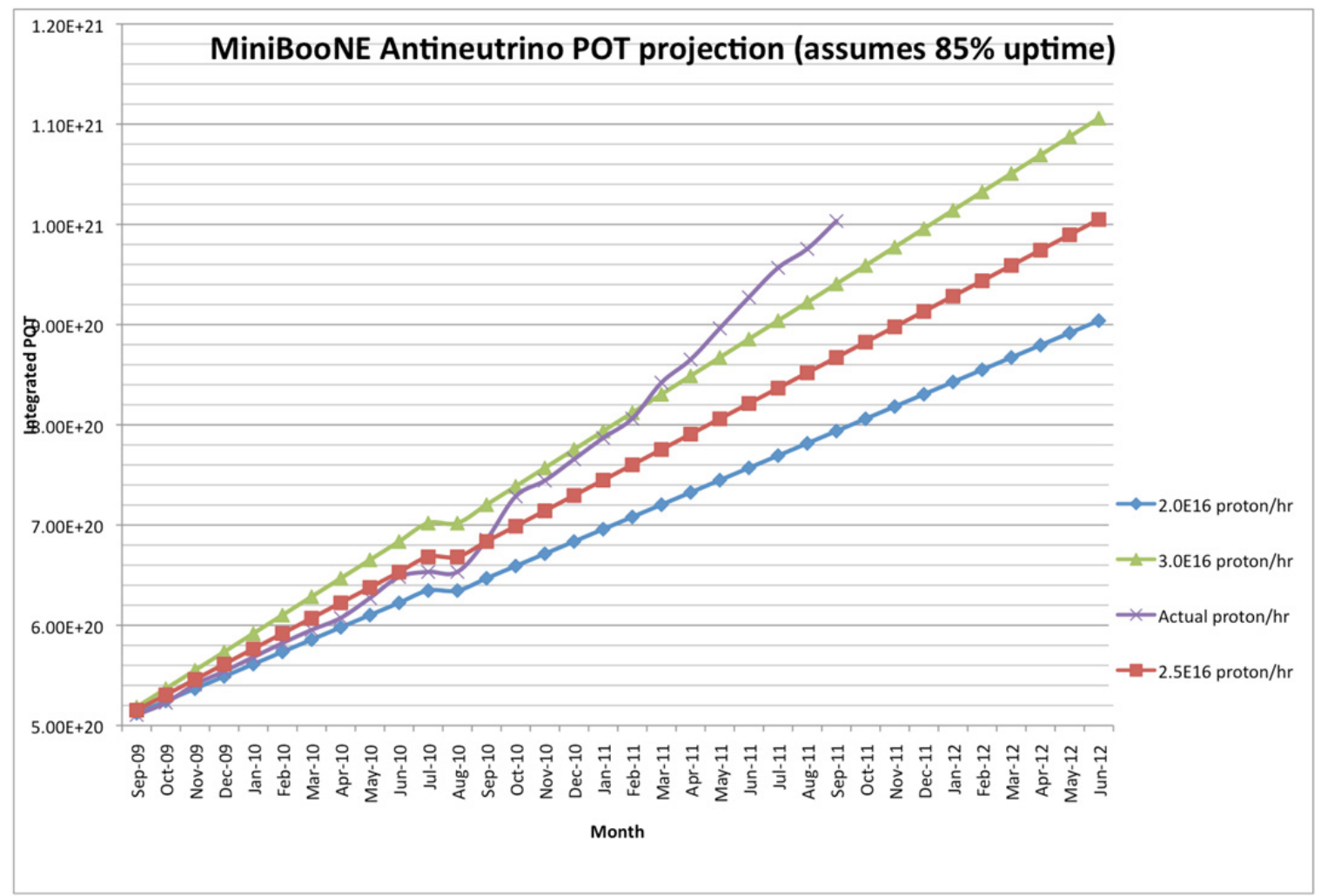

Figure 19. MiniBooNE actual integrated POT and projection for various beam rates. At the end of FY 2011 a total of $1.01 \times 10^{21}$ POT had been delivered in antineutrino mode. At recent rates MiniBooNE should reach about $1.2 \times 10^{21}$ POT by the long shutdown in 2012 .

An important aspect of the continued running of MiniBooNE is the reliability of the beamline and horn. For the FY 2011 antineutrino run period, the combined beamline and detector uptime was over $85 \%$. The most crucial element, the horn, operated for over 355 million pulses, surpassing the first horn, which failed seven years ago with 94 million pulses. Both these numbers were world records at the respective times. This second horn recently developed a cooling water leak and ground current trips. The Laboratory was able to mitigate the horn trips by draining the water catch pan, and the Booster Neutrino Beam continued to run with reduced cooling. After a week, the leak, caused by blockage due to a failed water pump, cleared up on its own. The Laboratory is taking steps to further isolate the water pump and also to replace it routinely before it fails. This should prevent future problems from this particular failure mode. In the unfortunate event of complete failure, a spare horn and target are available. It would require four to six weeks to install the replacement system.

Finally, the stability of the beamline and detector is demonstrated in Figs. 20 and 21, which show the number of neutrinos/POT and the muon neutrino energy over time. Aside from a short period with a beamline absorber problem in early 2006, the neutrino/POT rates have been constant. Also, detailed checks of neutrino energy response shows remarkable stability. This clearly indicates that both the beamline and detector are stable over time, which is crucial for long term running. 


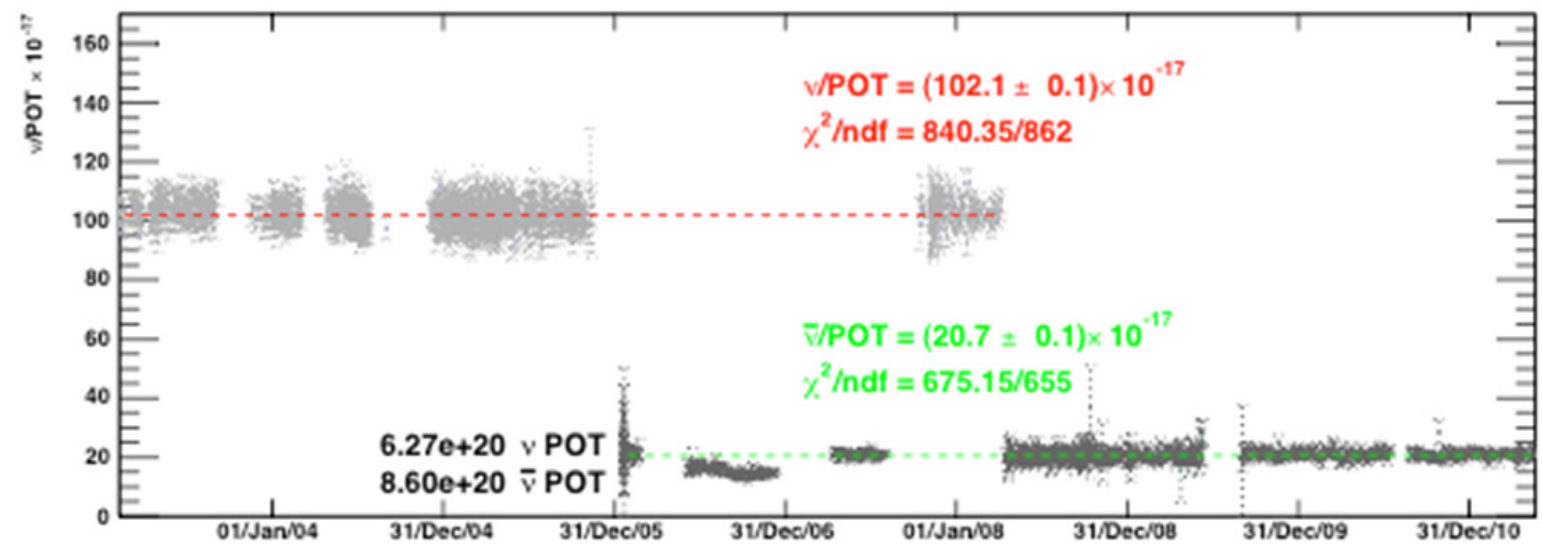

Figure 20. Neutrinos/POT for both neutrino and antineutrino running. The overall stability is excellent as shown by the $\mathrm{X}^{2} /$ ndf near one. The antineutrino mode does not include the absorber problem data in early 2006. The factor-of-five difference between neutrino and antineutrino modes is due to the reduced flux and cross section which makes taking high statistics antineutrino data samples difficult and time consuming.

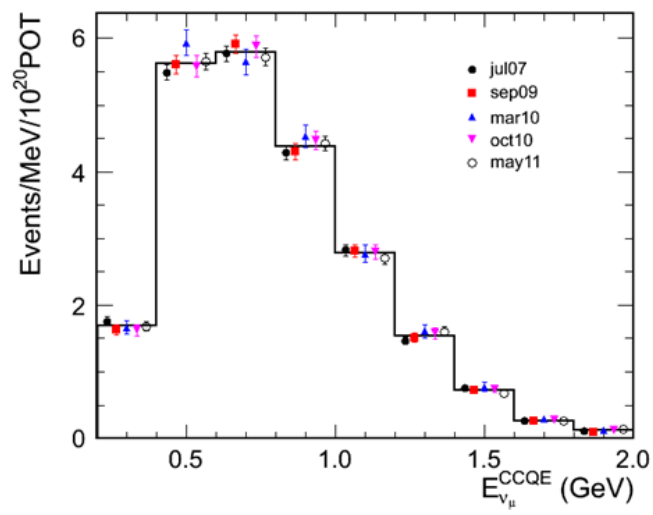

Figure 21. The reconstructed muon neutrino energy for different time periods. Very little difference can be seen, indicating that the production and reconstruction of the neutrinos has not changed over time.

Fixed-Target Switchyard 120 GeV (SY120) and MTest (A. K. Soha, R. Coleman, W. Kissel, C. Moore, E. Ramberg)

\section{Switchyard $120 \mathrm{GeV}$ Extraction from Main Injector in FY 2011}

The Fermilab test beam originates from the resonant extraction of one Booster batch inside the Main Injector (MI). This batch usually consists of 20-60 rf 'buckets', with buckets separated by $19 \mathrm{nsec}$. Thus the batch is about 1 microsecond long. The time required for a particle to transit the full circumference of the MI is about 11 microseconds. The batch is accelerated to $120 \mathrm{GeV}$, circulated around the MI, and is slowly extracted over a time period of a few seconds as a quadrupole gradually increases in field strength. 
The length and duty cycle of the spill is determined by the Accelerator Division (AD), with guidance from the Program Planning Office. For FY 2011, there was a single 4-second-long spill per minute, for a maximum of up to 14 hours per day. The AD has set up a procedure for changing from this four-second spill to a one-second spill. The shorter spill can then be delivered more frequently for commissioning purposes, and for those groups which are limited by dataacquisition buffers. The number of SY 120 pulses with beam in FY 2011 was 115,493, compared to 91,487 in FY 2010.

In addition to the 4-second-long spill, a "ping" beam extraction from the Main Injector is available, in which the extraction quadrupole is brought close to its nominal strength, and then pulsed with current over a very short $(1-5 \mathrm{msec})$ period. A large fraction of the beam then spills out in that short period. This can then be repeated at periodic intervals as the Main Injector holds its flattop. If the ping is $1 \mathrm{msec}$ long and the interval between pings is $200 \mathrm{msec}$, this replicates the macroscopic duty cycle of the proposed International Linear Collider (ILC). This makes Fermilab's MTest the only facility able to deliver high-energy particles with this structure. Figure 22 shows oscilloscope traces for this pinged extraction.

\section{First Pings to MTest}

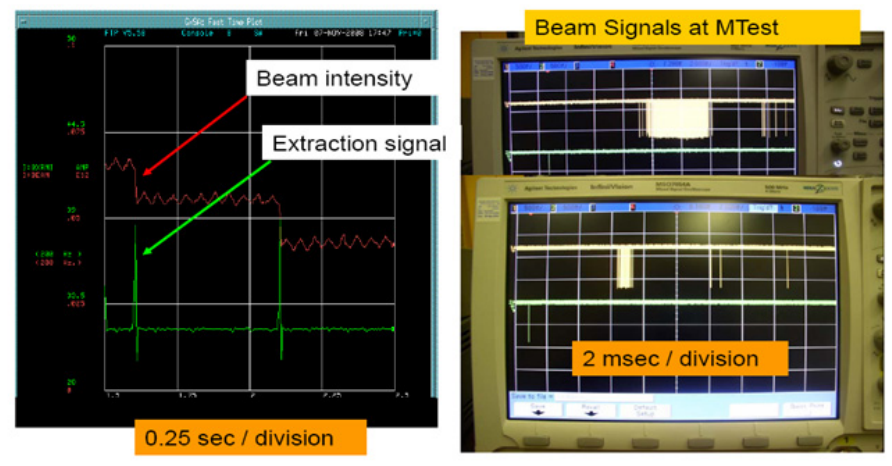

Figure 22. Two scope traces of scintillator hits during pinged MI extraction.

The extracted $120 \mathrm{GeV}$ primary proton beam has an approximate $0.3 \%$ momentum spread, and can be focused to a $7 \mathrm{~mm}$ rms spot size at the user facility (described below). In addition to delivering primary beam to users, the facility can target this primary beam on a secondary target and deliver lower-energy beams in the energy range of $1-66 \mathrm{GeV}$. There is a $1 \%$ to $10 \%$ momentum spread with approximately $2-5 \mathrm{~cm}$ rms spot size for secondary beams of lower momenta.

The MTest secondary beamline is optimized for low-energy particle production using a target located close to the user facility in order to minimize the loss of useful beam due to decays of low-energy mesons. The ILC CALICE calorimeter group (T-978) was particularly interested in the low-energy pion capability. In FY 2011, a number of groups utilized this secondary-beam mode. Tunes for the beamline were optimized for a variety of energies from 1 to $32 \mathrm{GeV} / \mathrm{c}$. In addition, it was observed that inserting a movable absorber located in the user area and tuning for positive $32 \mathrm{GeV} / \mathrm{c}$ secondary beam maximizes the rate ( $\mathrm{kHz}$ level) of a wide-band muon beam. 


\section{The Fermilab Test Beam Facility}

The Fermilab Test Beam Facility (FTBF) gives users from around the world an opportunity to set up their particle detectors in a variety of particle-beams. A plan view of the facility is shown in Fig. 23. The web-site URL for the facility is www-ppd.fnal.gov/FTBF.

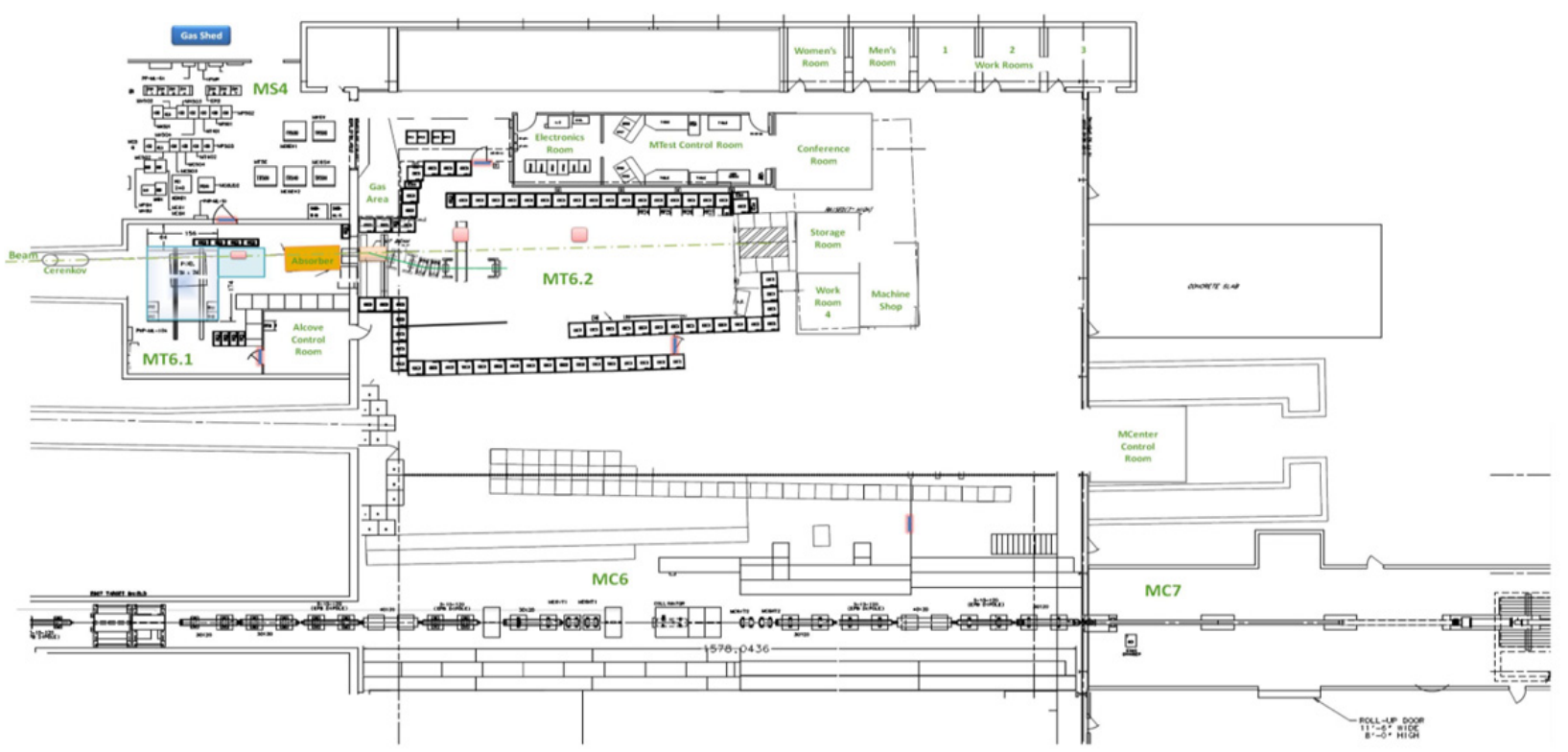

Figure 23. Plan view of the Fermilab Test Beam Facility.

\section{Research Performed at the FTBF in FY 2011}

Each test-beam experiment is required to prepare a Memorandum of Understanding with the Laboratory, in which the beam, infrastructure, and safety requirements are spelled out in detail. Six new experiments were approved and took data during FY 2011, and an additional seven experiments returned from previous years to take more data in FY 2011. These thirteen experiments are listed in Table IV, and represent 223 collaborators from 60 institutions in 14 countries. The chart in Figure 24 shows the growth in these numbers over the last 5 years.

Table IV. Test Beam experiments performed in FY 2011.

\begin{tabular}{|l|l|}
\hline \multicolumn{1}{|c|}{ Test } & Description \\
\hline \multicolumn{2}{|c|}{} \\
\hline T-932 & Diamond Detector \\
\hline T-953 & U of Iowa Calorimetry \\
\hline T-978 & CALICE \\
\hline T-979 & Fast Timing Counters for PSEC \\
\hline T-992 & SLHC sensor tests \\
\hline T-994 & JASMIN \\
\hline T-1004 & Dual Readout Calorimetry \\
\hline T-1008 & SuperB Prototype \\
\hline T-1010 & GEM Chamber Characteristics Test \\
\hline T-1011 & SLHC sensor tests \\
\hline T-1012 & TAUWER Test \\
\hline
\end{tabular}




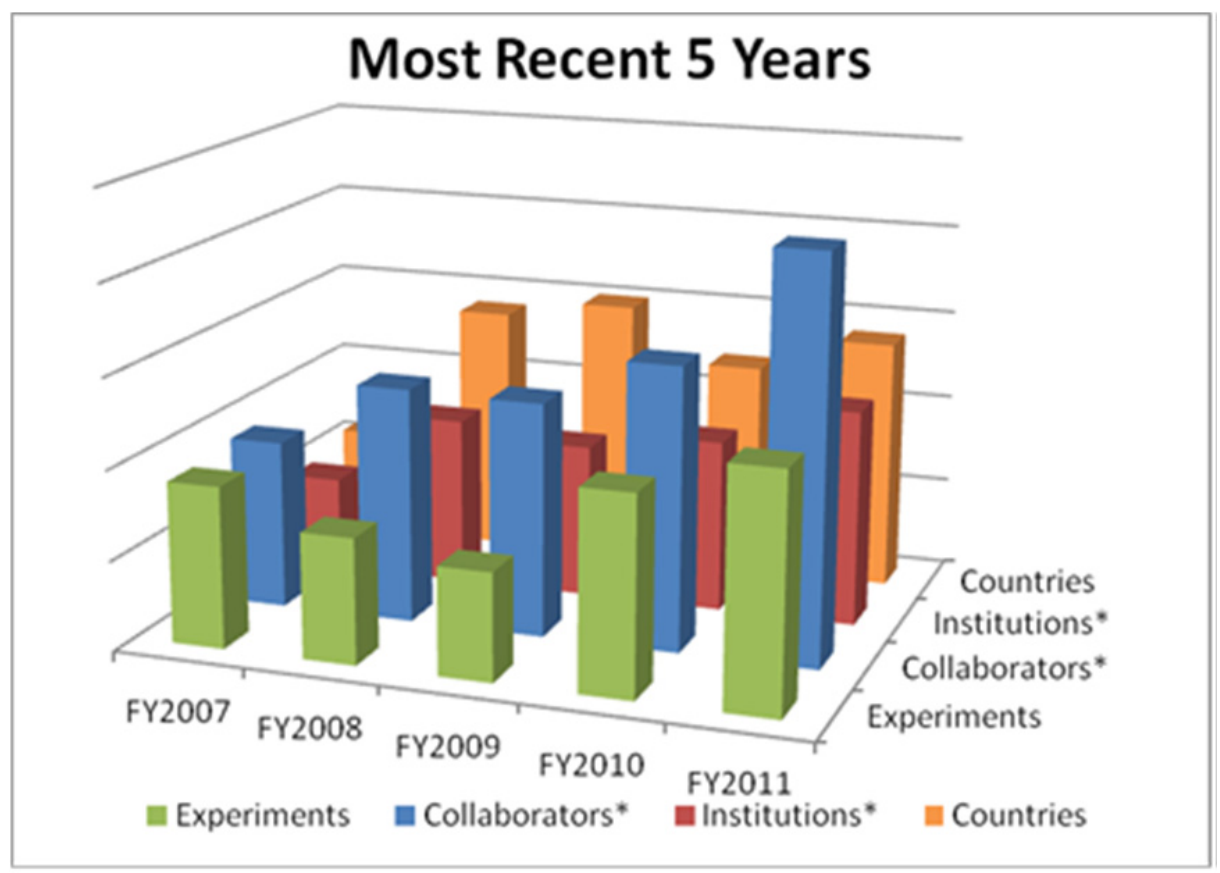

Figure 24: Growth in number of experiments, collaborators, institutions and countries served by the FTBF over the past 5 years. *Number of Collaborators has been scaled to fit on plot. *Number of Institutuions has been scaled to fit on plot.

The experiments installed equipment or took data $91 \%$ of the time for a total of 44.5 weeks, out of the 49 weeks with beam available during the year, including 4 weeks of double occupancy (two experiments taking data at once). In addition, FTBF hosted a 3 day workshop on Detector R\&D in early October. Weekly facility usage since 2005 (when the facility started taking beam) is shown in Figure 25.

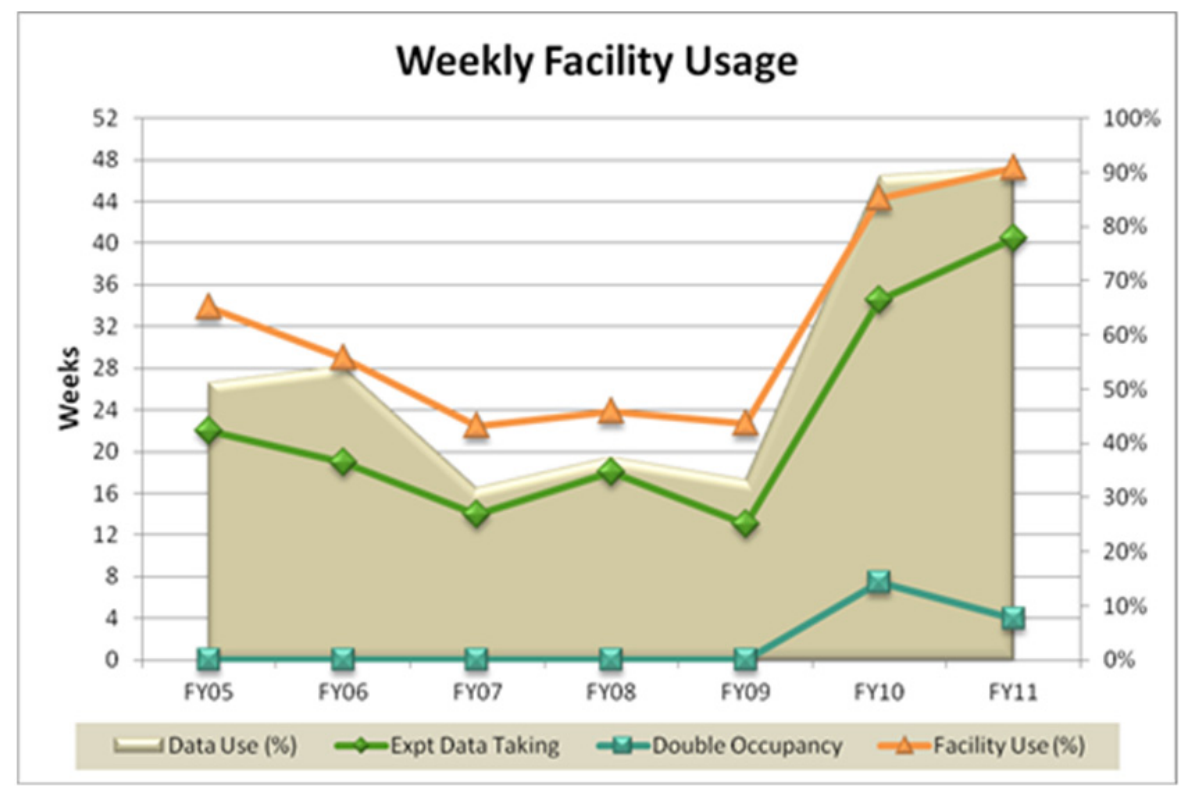

Figure 25: A week with an experiment taking data is shown in green, a week with 2 experiments taking data is shown in blue. Data Use is a percentage combining those numbers with respect to the number of weeks beam was available. Facility Use (orange) includes Data Use and Installation time with respect to the number of weeks available. 


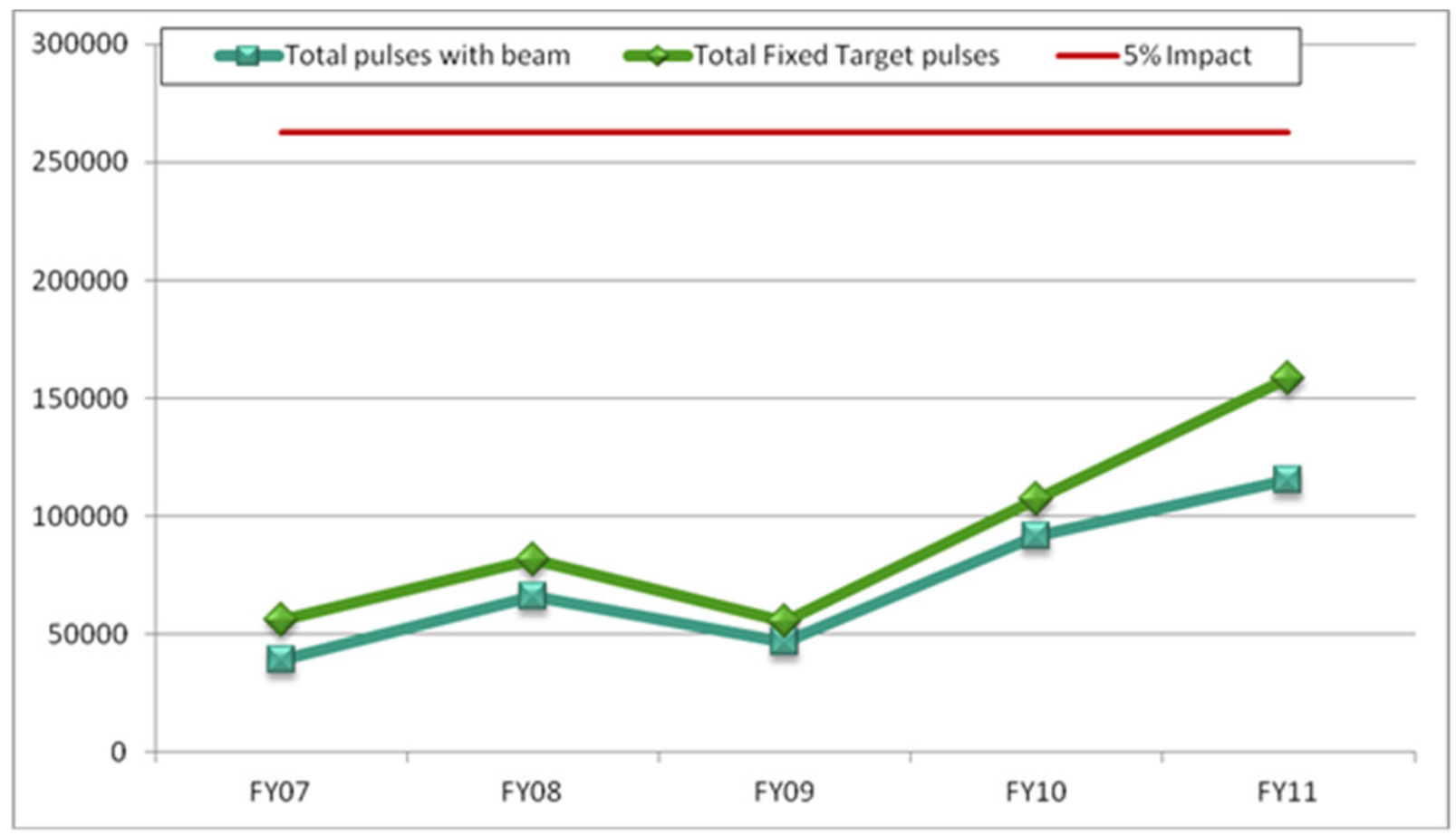

Figure 26: Fixed target pulses, to FTBF with and without beam over the past 5 years

During the 44.5 weeks of data taking, a total of 158,708 FTBF beam cycles occurred, 115,493 of which had beam. The FTBF used a total of $\mathrm{f} 3.60 \times 10^{15}$ protons, but many of the experiments requested very low intensity running. The chart in Fig. 26 shows the numbers of beam cycles per year over the last 5 years, and depicts how FTBF operations have been well below the 5\% impact limit set by the Director. 